\title{
The evolution of compact massive quiescent and star-forming galaxies derived from the $R_{\mathrm{e}}-R_{\mathrm{h}}$ and $M_{\mathrm{star}}-M_{\mathrm{h}}$ relations
}

L. Zanisi, ${ }^{1,2 \star ~}{ }^{\text {F. Shankar }}{ }^{\circledR},{ }^{1 \star}$ H. Fu, ${ }^{1}$ A. Rodriguez-Puebla ${ }^{3}$ V. Avila-Reese ${ }^{\circledR},{ }^{3}$ A. Faisst, ${ }^{4}$ E. Daddi, ${ }^{5}$ L. Boco, ${ }^{6}$ A. Lapi, ${ }^{6,7}$ M. Giavalisco ${ }^{\circledR},{ }^{8}$ P. Saracco ${ }^{\circledR},{ }^{9}$ F. Buitrago ${ }^{\circledR},{ }^{10,11}$ M. Huertas-Company ${ }^{(0)},{ }^{12,13}$ A. Puglisi ${ }^{\circledR 14}$ and A. Dekel ${ }^{15}$

${ }^{1}$ Department of Physics and Astronomy, University of Southampton, Highfield SO17 1BJ, UK

${ }^{2}$ DISCnet Centre for Doctoral Training, University of Southampton, Highfield SO17 $1 B J, U K$

${ }^{3}$ Instituto de Astronomía, Universidad Nacional Autónoma de México, A. P. 70-264, 04510, México, D.F., México

${ }^{4}$ PAC, California Institute of Technology, 1200 E California Boulevard, Pasadena, CA 91125, USA

${ }^{5}$ CEA, IRFU, DAp, AIM, Universitè Paris-Saclay, Universitè Paris Diderot, Sorbonne Paris Citè, CNRS, F-91191 Gif-sur-Yvette, France

${ }^{6}$ SISSA, Via Bonomea 265, I-34136 Trieste, Italy

${ }^{7}$ IFPU - Institute for fundamental physics of the Universe, Via Beirut 2, I-34014 Trieste, Italy

${ }^{8}$ Department of Astronomy, University of Massachusetts Amherst, 710 N. Pleasant St, Amherst, MA 01003, USA

${ }^{9}$ INAF - Osservatorio Astronomico di Brera, via Brera 28, I-20121 Milano, Italy

${ }^{10}$ Departamento de Física Teórica, Atómica y Óptica, Universidad de Valladolid, E-47011 Valladolid, Spain

${ }^{11}$ Instituto de Astrofísica e Ciências do Espaço, Universidade de Lisboa, OAL, Tapada da Ajuda, PT1349-018 Lisbon, Portugal

${ }^{12}$ Instituto de Astrofísica de Canarias (IAC); Departamento de Astrofísica, Universidad de La Laguna (ULL), E-38200 La Laguna, Spain

${ }^{13}$ LERMA, Observatoire de Paris, CNRS, PSL, Université Paris Diderot, F-75014 Paris, France

${ }^{14}$ Center for Extragalactic Astronomy, Durham University, South Road, Durham DH1 3LE, UK

${ }^{15}$ Racah Institute of Physics, The Hebrew University, Jerusalem 91904, Israel

Accepted 2021 May 17. Received 2021 March 27; in original form 2020 November 10

\begin{abstract}
The mean size (effective radius $R_{\mathrm{e}}$ ) of massive galaxies (MGs; $M_{\mathrm{star}}>10^{11.2} \mathrm{M}_{\odot}$ ) is observed to increase steadily with cosmic time. It is still unclear whether this trend originates from the size growth of individual galaxies (via, e.g. mergers and/or AGN feedback) or from the inclusion of larger galaxies entering the selection at later epochs (progenitor bias). We here build a data-driven, flexible theoretical framework to probe the structural evolution of MGs. We assign galaxies to dark matter haloes via stellar mass-halo mass (SMHM) relations with varying high-mass slopes and scatters $\sigma_{\text {SMHM }}$ in stellar mass at fixed halo mass, and assign sizes to galaxies using an empirically motivated, constant and linear relationship between $R_{\mathrm{e}}$ and the host dark matter halo radius $R_{\mathrm{h}}$. We find that (1) the fast mean size growth of MGs is well reproduced independently of the shape of the input SMHM relation; (2) the numbers of compact MGs grow steadily until $z \gtrsim 2$ and fall off at lower redshifts, suggesting a lesser role of progenitor bias at later epochs; (3) a time-independent scatter $\sigma_{\text {SMHM }}$ is consistent with a scenario in which compact star-forming MGs transition into quiescent MGs in a few $10^{8} \mathrm{yr}$ with a negligible structural evolution during the compact phase, while a scatter increasing at high redshift implies significant size growth during the star-forming phase. A robust measurement of the size function of MGs at high redshift can set strong constraints on the scatter of the SMHM relation and, by extension, on models of galaxy evolution.
\end{abstract}

Key words: galaxies: abundances - galaxies: fundamental parameters - galaxies: high-redshift-galaxies: star formation.

\section{INTRODUCTION}

There is now substantial evidence that galaxies of a given stellar mass are smaller at higher redshift than in the local Universe (e.g. Daddi et al. 2005; Trujillo et al. 2007; Buitrago et al. 2008; van Dokkum et al. 2010, 2015; Cassata et al. 2011; Cimatti, Nipoti \& Cassata 2012; Newman et al. 2012; Huertas-Company et al. 2013; Kawamata et al. 2015; Shibuya, Ouchi \& Harikane 2015). The size evolution of the galaxy population in a given stellar mass bin is well

^E-mail: 1.zanisi@soton.ac.uk (LZ); f.shankar@soton.ac.uk (FS) fitted by a relation of the type

$R_{\mathrm{e}}(z) \propto(1+z)^{-\alpha}$,

where $R_{\mathrm{e}}$ is defined as the radius that encloses half of the galaxy light (see e.g. van der Wel et al. 2014 for a different fitting function). It is found that in general star-forming galaxies follow shallower trends (lower values of $\alpha$ ) than quiescent galaxies (e.g. van der Wel et al. 2014). Notably, the size growth rate of star-forming galaxies increases with stellar mass, becoming comparable to that of quiescent galaxies (with $\alpha \sim 1$ ) for $M_{\text {star }}>10^{11.2} \mathrm{M}_{\odot}$ (Faisst et al. 2017; Mowla et al. 2019b). Moreover, in this mass regime, as pointed out in several studies (e.g. Bernardi et al. 2011a,b; Cappellari 2016), 
the behaviour of the scaling relations and the stellar kinematics differ from that of less massive galaxies (MGs). Thus, the mass scale $M_{\text {star }} \approx 10^{11.2} \mathrm{M}_{\odot}$ is critical to understanding galaxy evolution. In this paper, we focus on the structural evolution of galaxies in this high mass regime, which we simply label in what follows as 'MGs'.

There is no consensus yet as to why MGs were a factor of 3 to 5 smaller in the past. Minor dry mergers have been invoked as an efficient channel to promote substantial size increase with relatively modest change in stellar mass (e.g. Naab, Johansson \& Ostriker 2009; Oser et al. 2010; Shankar et al. 2013; van Dokkum et al. 2015) to accommodate for the limited evolution in the high-mass end of the stellar mass function (SMF) since $z \sim 1.5$ (e.g. Andreon 2013; Muzzin et al. 2013; McDermid et al. 2015; Kawinwanichakij et al. 2020). However, the rate of minor dry mergers may not be sufficient by themselves to account for the entire size evolution of MGs through cosmic time (Newman et al. 2012; Nipoti et al. 2009, 2012). More generally, the exact contribution of dry mergers to the mass assembly of MGs is still a matter of intense debate among both theoretical studies (e.g. De Lucia \& Blaizot 2007; Hopkins et al. 2010b; Wilman et al. 2013; Rodriguez-Gomez et al. 2015; Qu et al. 2017; Tacchella et al. 2019; Grylls, Shankar \& Conselice 2020b; O'Leary et al. 2021) and observational works (e.g. Man, Zirm \& Toft 2016; Mundy et al. 2017; Mantha et al. 2018; Duncan et al. 2019).

It has often been debated in the literature whether the size evolution of galaxies of a given stellar mass stems from the size growth of individual galaxies or it is a consequence of a 'population effect' where newly formed, larger galaxies enter the mass selection at later epochs thus increasing the mean size distribution (e.g. Carollo et al. 2013; Shankar et al. 2015; Gargiulo et al. 2017). This 'progenitor bias' effect (van Dokkum \& Franx 1996) has been usually invoked to explain the size evolution of passive galaxies with $M_{\text {star }}<10^{11} \mathrm{M}_{\odot}$ (e.g. Fagioli et al. 2016; Faisst et al. 2017). Most studies agree on the lesser role of progenitor bias in the size evolution of MGs at $z \lesssim 1$, in favour of a more predominant role of (dry) mergers in increasing the sizes of individual galaxies (e.g. Saglia et al. 2010; Carollo et al. 2013; van der Wel et al. 2014; Fagioli et al. 2016; Faisst et al. 2017; but see also Gargiulo et al. 2017). In particular, the disappearance of compact (e.g. Cassata et al. 2011; Barro et al. 2013) galaxies as the Universe ages is interpreted as a sign that they must have grown in size individually (van der Wel et al. 2014) while a constant abundance of compact galaxies implies that progenitor bias dominates the size growth (Saracco, Longhetti \& Gargiulo 2010; Gargiulo et al. 2016, 2017). In this respect, the full distribution of galaxy sizes at fixed stellar mass, i.e. the size function $\phi\left(R_{\mathrm{e}} \mid M_{\text {star }}\right)$, is an invaluable tool to disentangle galaxy evolution scenarios, providing simultaneous information on the mean size $R_{\mathrm{e}}$ and the number density of compact galaxies (e.g. Shankar et al. 2010; Carollo et al. 2013; Zanisi et al. 2020, hereafter Z20).

In addition to mergers and progenitor bias, active galactic nuclei (AGNs) feedback during the compact star-forming stages of the evolution of MGs (both in a submm-FIR phase, e.g. Barro et al. 2016, and an optical 'blue nugget' phase, e.g. Martig et al. 2009; Damjanov et al. 2011; Barro et al. 2013; Fang et al. 2013; Zolotov et al. 2015; Tacchella et al. 2016, which are potentially linked in an evolutionary sequence, e.g. Gómez-Guijarro et al. 2019; Puglisi et al. 2021) may also contribute to both size growth and quenching (Fan et al. 2008, 2010; Kocevski et al. 2017; Lapi et al. 2018a; van der Vlugt \& Costa 2019), and the relative evolution of compact starforming and quiescent galaxies can provide tight constraints on these processes, as we will further discuss below.
Due to their flexibility and relatively lower number of free parameters, semi-empirical models have become a popular route to study the mass assembly, star formation, and merger histories of galaxies (e.g. Conroy \& Wechsler 2009; Hopkins et al. 2010a,b; Behroozi, Wechsler \& Conroy 2013; Moster, Naab \& White 2013; Shankar et al. 2013; Gu, Conroy \& Behroozi 2016; Matthee et al. 2017; Rodríguez-Puebla et al. 2017; Tinker 2017; Lapi et al. 2018a; Grylls et al. 2020a,b; Chen et al. 2020; O'Leary et al. 2021). The main ingredient in semi-empirical models is the input stellar mass-halo mass (SMHM) relation, which is extracted from the cumulative equivalence between the number densities of the stellar mass and (sub)halo mass functions (e.g. Shankar et al. 2006; Vale \& Ostriker 2006; Dutton et al. 2010; Firmani \& Avila-Reese 2010; Guo et al. 2011; Leauthaud et al. 2012; Rodríguez-Puebla, AvilaReese \& Drory 2013; Mandelbaum et al. 2016; Kravtsov, Vikhlinin \& Meshcheryakov 2018; Moster, Naab \& White 2018; Pillepich et al. 2018; Behroozi et al. 2019; Erfanianfar et al. 2019). However, the systematic uncertainties in the input data, most notably in the SMF, ${ }^{1}$ have yielded discrepant results along the years. For example, some groups (e.g. Behroozi et al. 2013; Moster et al. 2013; RodríguezPuebla et al. 2017) proposed a shallower high-mass slope in the SMHM relation, while others have argued in favour of a steeper slope (e.g. Shankar et al. 2014; Tinker et al. 2017; Kravtsov et al. 2018; Grylls et al. 2019). In turn, the high-mass end of the SMHM relation plays a crucial role in, e.g. the number of galaxy pairs, and thus on galaxy merger rates (Grylls et al. 2020b; O'Leary et al. 2021).

Some works also highlighted a further correlation between the size of a galaxy, $R_{\mathrm{e}}$, and that of its host dark matter halo, $R_{\mathrm{h}}$ (e.g. Fall 1983; Mo, Mao \& White 1998; Kravtsov 2013; Huang et al. 2017; Desmond et al. 2017; Somerville et al. 2018; Lapi, Salucci \& Danese 2018b; Hearin et al. 2019; Mowla et al. 2019a; Z20, but see also Desmond 2017). When coupled together in a semi-empirical model, the SMHM and $R_{\mathrm{e}}-R_{\mathrm{h}}$ relations become powerful tools to simultaneously probe the mass and structural evolution of galaxies in a full cosmological context. For instance, Somerville et al. 2018 found a non-trivial redshift and stellar mass dependence of the mean $R_{\mathrm{e}}-R_{\mathrm{h}}$ relation at $M_{\text {star }} \lesssim 10^{11.2} \mathrm{M}_{\odot} . \mathrm{Z} 20$, found that, in the MGs regime and at $z \sim 0.1$, the $R_{\mathrm{e}}-R_{\mathrm{h}}$ relation must be very tight with a total scatter (inclusive of observational statistical uncertainties) of only $\approx 0.1 \mathrm{dex},{ }^{2}$ Stringer et al. (2014, hereafter S14) combined the Moster et al. (2013) SMHM relation and a constant $R_{\mathrm{e}}-R_{\mathrm{h}}$ relation to build a semi-empirical model which proved capable to reproduce the size evolution of MGs in the COSMOS field (Huertas-Company et al. 2013). S14 attributed the mean size growth of the population of MGs at $z \lesssim 2$ to a cosmological effect for which (i) the size of the host dark matter haloes of a given mass become larger as the Universe expands and its density decreases and (ii) MGs of the same mass form in more massive, extended dark matter haloes at

${ }^{1}$ Ultimately, the reasons for these discrepancies are thought to originate from the way stellar masses are estimated. The initial mass function, dust attenuation curve, stellar population synthesis models and assumed star formation histories, the inclusion of intra-cluster light and even the photometry choice and background subtraction algorithms all contribute to the various determinations of the stellar mass functions found in the literature (see e.g. Bernardi et al. 2010, 2013, 2016, 2017; Kravtsov et al. 2018; Guarnieri et al. 2019; Kawinwanichakij et al. 2020; Leja et al. 2020; Lower et al. 2020 among many others), which result in different estimates of the SMHM relation (e.g. Shankar et al. 2017).

${ }^{2}$ This was necessary to reproduce the full size distribution of early- and latetype MGs in the local Universe as measured in the Sloan digital Sky Survey (SDSS). 

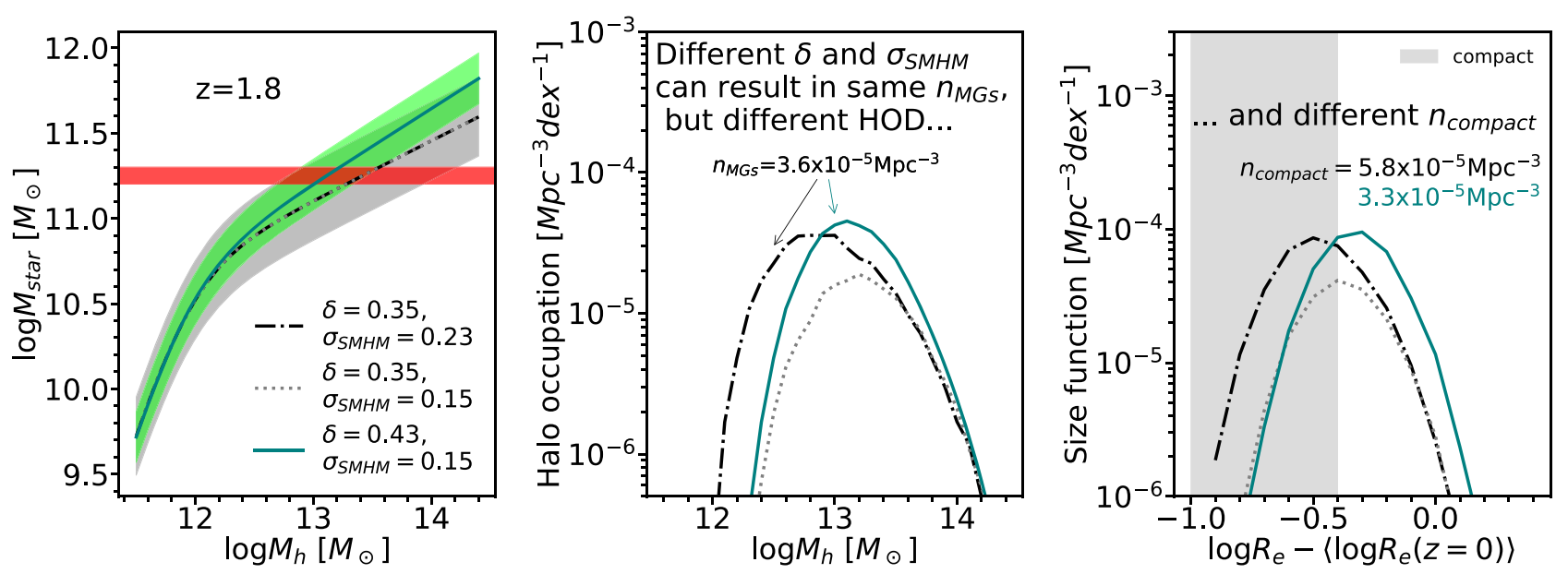

Figure 1. The effect of different SMHM relations on the halo occupation distribution (HOD) of MGs and the size functions implied by a linear $R_{\mathrm{e}}-R_{\mathrm{h}}$ relation (equation 4). We show that SMHM relations with different high-mass slope $\delta$ and scatter $\sigma_{\text {SMHM }}$ (shown as shaded areas in the left-hand panel) can produce the same number density for MGs (the mass threshold for MGs is shown as a red horizontal line). However, the HOD for the two models is remarkably different (central panel). This translates in very different size functions. In particular, the number density of compact galaxies differs by almost a factor of 2 (we use the Cassata et al. 2013 definition of compactness, i.e. 0.4 dex below the $z=0$ mean size, against which we calibrated the two models following Appendix B). The model indicated with dashed grey lines is shown to help appreciate the effect of a lower $\sigma_{\text {SMHM }}$, at fixed $\delta$, on the halo occupation distribution and on the size function (compare to the dot-dashed model). Although we show results only at a given redshift as an example, the same arguments apply at any epoch for some choices of $\delta$ and $\sigma_{\mathrm{SMHM}}$.

lower redshift. While these results are encouraging, the effects of assuming a different SMHM relation in the framework outlined by S14 have remained relatively unexplored. The only notable exception is the work by Mowla et al. (2019a), where, however, only the shape of the SMHM was considered and not its scatter, $\sigma_{\mathrm{SMHM}}$. The parameter $\sigma_{\text {SMHM }}$ is notoriously degenerate with the high-mass slope of the SMHM relation in retrieving the number density of MGs (Shankar et al. 2014; Wechsler \& Tinker 2018). As we will show, instead, $\sigma_{\text {SMHM }}$ has a seizable effect on the number density of the population of compact galaxies, and therefore it is a significant novelty that we include in the semi-empirical framework set out in S14.

In this paper, we put forward a phenomenological, transparent methodology to probe the size evolution of MGs that expands on the works by S14, Somerville et al. (2018), and Mowla et al. (2019a). Following the approach of Grylls et al. (2020b), we build mock catalogues of galaxies in dark matter haloes using data-driven toy models where SMHM relations with different shapes and dispersion are coupled with linear $R_{\mathrm{e}}-R_{\mathrm{h}}$ relations characterized by different normalizations. The main objective of this paper is to probe the impact of varying the input SMHM relation and its dispersion $\sigma_{\mathrm{SMHM}}$ on: (1) the mean size evolution of MGs, (2) the full size function of MGs across cosmic time, and (3) the number density of compact MGs. The latter point is particularly original and powerful as the time dependence of the number density of compact MGs is closely linked to progenitor bias: less compact galaxies at fixed stellar mass would be observed at later epochs if they grow in size via, e.g. mergers. We will show, in particular, that the scatter in the SMHM relation plays a major role in setting the number density of compact MGs, allowing to break the degeneracy between the scatter and the high-mass slope of the SMHM (see e.g. Fig. 1). Our present work lays out an effective strategy to unveil the evolutionary pathways of MGs by exploiting the increased statistics of MGs that will become available from future observations. Data for MGs are in fact at present quite sparse and uncertain at $z \gtrsim 1$ (e.g. Kawinwanichakij et al. 2020), and effective radii have been measured for only a handful of MGs at $z \gtrsim 2$ (e.g.
Faisst et al. 2017; Kubo et al. 2017; Patel et al. 2017; Mowla et al. 2019b; Lustig et al. 2021; Stockmann et al. 2021).

The outline of the paper is as follows. In Section 2, we provide the backbone of our framework. In Section 3.1, we explore the role of the scatter in the SMHM relation and we present toy models inspired to these findings in Section 3.2. In Sections 3.3, 3.4, and 3.5, we show how the different SMHM implied by the toy models results in a range of possible determinations of the size distributions of MGs. We give an interpretation of the evolutionary pathways of MGs implied by the different toy models in Section 4.1, and we show how our framework can be used to constrain the shape and scatter of the SMHM relation in Section 4.2. In Section 4.3, we discuss the possibility that the SMHM and $R_{\mathrm{e}}-R_{\mathrm{h}}$ relations are correlated, and in Section 4.4 we discuss an extension of the model of the $R_{\mathrm{e}}-R_{\mathrm{h}}$ connection based on halo concentration, and discuss its limitations and strengths. Finally, we draw our conclusions in Section 5. Further material is available in the Appendices.

\section{METHODS}

\subsection{The galaxy-halo connection}

To study the size distribution and evolution of MGs $\left(M_{\text {star }}>\right.$ $10^{11.2} \mathrm{M}_{\odot}$ ), at any redshift of interest we build a catalogue of dark matter haloes with mass $M_{\mathrm{h}}$ and size $R_{\mathrm{h}}$, to which we assign a stellar mass $M_{\text {star }}$ (via an input SMHM relation) and an effective radius $R_{\mathrm{e}}$ (via a $R_{\mathrm{e}}-R_{\mathrm{h}}$ relation). The detailed modelling approach that we use here closely follows the one outlined in Z20, which we briefly summarize below for convenience: ${ }^{3}$

(i) We use the Despali et al. (2016) halo mass function to obtain large catalogues of dark matter haloes at all the redshifts of interest. Note that the Despali et al. (2016) halo mass function is defined

\footnotetext{
${ }^{3}$ We make extensive use of the colossus Python package (Diemer 2018).
} 
for central galaxies only, as it does not include subhaloes (e.g. van den Bosch et al. 2014). Thus, all MGs in this study are modelled as central galaxies. ${ }^{4}$ In this paper, we model dark matter haloes with a Navarro, Frenk \& White (1996) density profile with scale radius $R_{\mathrm{s}}$,

$\rho(r) \propto \frac{1}{\frac{r}{R_{\mathrm{S}}}\left[1+\frac{r}{R_{\mathrm{S}}}\right]^{2}}$,

and with $R_{\mathrm{h}}=c R_{\mathrm{s}}$ defining the concentration parameter $c . R_{\mathrm{h}}$ is the dark matter halo radius,

$R_{\mathrm{h}}=\left(\frac{3 M_{\mathrm{h}}}{4 \pi \Delta \rho_{\Delta}}\right)^{\frac{1}{3}}$

where $\Delta$ is the virial overdensity with respect to the cosmological critical density (Bryan \& Norman 1998). Both $\Delta$ and $\rho_{\Delta}$ are decreasing functions of cosmic time (e.g. Mo, van den Bosch \& White 2010). Thus, a dark matter halo of a given mass has a smaller size at higher redshift, owing to the higher density of the Universe.

(ii) We model the link between galaxies and dark matter via the $\mathrm{SMHM}$ relation. The mean $\mathrm{SMHM} \equiv M_{\text {star }}\left(M_{\mathrm{h}}\right)$ is a monotonically increasing function of halo mass. We include a lognormal scatter $\sigma_{\text {SMHM }}$ at fixed halo mass that takes into account both the intrinsic dispersion $\sigma_{\text {intr }}$ in the relation and the uncertainty in stellar mass estimates $\sigma_{*}$ (i.e. $\sigma_{\mathrm{SMHM}}^{2}=\sigma_{\text {intr }}^{2}+\sigma_{*}^{2}$; see Behroozi et al. 2013; Shankar et al. 2014; Rodríguez-Puebla et al. 2017; Tinker et al. 2017). In the next section, we will present 'toy' SMHM relations, which vary in both shape and dispersion, to probe their impact on our galaxy mocks and on their size distributions at different epochs. In particular, we will focus on the slope above the knee of the SMHM relation, $\delta$ (see Fig. 1), which is the parameter in the SMHM relation controlling the number density of MGs at a given dispersion. The precise value of $\delta$, or better of the underlying abundances of MGs in the local and high redshift Universe, still suffer from substantial systematic uncertainties and that are hotly debated in the literature (see Section 1).

(iii) We assign a half-light radius $R_{\mathrm{e}}$ to each galaxy according to the ansatz:

$R_{\mathrm{e}}=A_{K} R_{\mathrm{h}}$,

which is based on the empirical findings by Kravtsov (2013), and that we call the $K 13$ model. Here, $A_{K}$ is the normalization which in principle may vary with halo mass, galaxy stellar mass and/or star formation activity (e.g. Huang et al. 2017; Somerville et al. 2018; Z20). We add to equation (4) an intrinsic lognormal scatter $\sigma_{K}$, which, as $A_{K}$, is a free parameter. We stress that, effectively, $R_{\mathrm{e}}$ is a function of $M_{\mathrm{h}}$, since there is a direct proportionality between halo mass and halo radius (see equation 3 ). While in the remainder of the paper we will mostly comment on the K13 model, in Section 4.4 we discuss another model of galaxy sizes in which the relation between virial radius and galaxy size is also mediated by the halo concentration (e.g. Desmond 2017; Jiang et al. 2019; Z20). Moreover, other definitions of galaxy sizes, such as $R_{80}$ (Miller et al. 2019) or $R_{1}$ (Trujillo, Chamba \& Knapen 2020), have been proposed to correlate to $R_{\mathrm{h}}$ equally well or even better than effective radius. We will discuss these models in Appendix E.

${ }^{4}$ In our model all MGs are considered central galaxies, since satellites are negligible in this extreme mass range (e.g. Peng et al. 2010). Using the Statistical Semi-Empirical Model STEEL (Grylls et al. 2019), we find that the satellite contribution to MGs is less than 20 per cent at $z \sim 0.1$, and it declines steeply at earlier times. The subdominant population of satellite MGs is studied in a companion paper (Z20).

\subsection{Quenching}

To provide a fair comparison to observations, which have so far always distinguished between star-forming and quiescent MGs (e.g. Mowla et al. 2019b), we need to include a recipe for quiescence in our galaxy mocks. To this purpose, following the empirical calibration of Rodríguez-Puebla et al. (2015) at $z \sim 0.1$, we assume that the probability of a galaxy being quenched in a dark matter halo of mass $M_{\mathrm{h}}$ is given by the fraction

$f_{\text {Quench }}\left(M_{\mathrm{h}}\right)=\frac{1}{b_{0}+\left[\mathcal{M}_{0} \times 10^{12} / M_{\mathrm{h}}\left(\mathrm{M}_{\odot}\right)\right]}$

with $b_{0} \sim 1$ and $\mathcal{M}_{0} \sim 0.68$ at $z \sim 0.1$. $f_{\text {Quench }}$ is a monotonically increasing function of halo mass, with a characteristic mass scale $\mathcal{M}_{0}$ above (below) which more (less) than 50 per cent of galaxies are quiescent (star-forming).

The fraction of quenched MGs is observed to evolve with redshift (e.g. Huertas-Company et al. 2016; Mowla et al. 2019b). While it is beyond the scope of this work to set specific constraints on the physical processes that drive quenching (see Somerville \& Davé 2015 for a review), we note that quenching is thought to be more likely to occur in more massive haloes at higher redshift (e.g. see the empirical models by Rodríguez-Puebla et al. 2017; Behroozi et al. 2019). In our model, this is achieved by replacing $\mathcal{M}_{0}$ with

$\mathcal{M}(z)=\mathcal{M}_{0}+(1+z)^{\mu}$,

where $\mu>0$ is a free parameter, which regulates the increase in characteristic quenching halo mass in the younger Universe. Fig. A1 shows examples of the evolution in $f_{\text {Quench }}$ for $\mu=1,3,5$. We note that quiescence is defined in the literature according to different methods (e.g. $1 \sigma$ below the main sequence, different cuts in the colour-colour planes, a hard cut in specific star formation rate, see e.g. Donnari et al. 2019) that can lead to different results (Sherman et al. 2020). Therefore, the value of $\mu$ will depend on the method assumed. For this reason, in the following we simply show different values of $\mu$, which we will adapt to the specific method used once the comparison data are fixed.

Quiescent and star-forming MGs of similar mass appear to grow in size at the same rate with redshift, with quiescent galaxies being systematically smaller at all times (Mowla et al. 2019b). We assume that the two populations live on two separate K13 relations. The normalizations $A_{K, S F}$ and $A_{K, Q}$, for star-forming and quenched MGs, respectively, are calibrated at $z \sim 0.1$ following Appendix B. Following Z20, we also assume that the scatters in the two K13 relations, $\sigma_{K, S F}$ and $\sigma_{K, Q}$, are equal to $0.1 \mathrm{dex}$. In the remainder of this paper, we assume that this value of $\sigma_{K}$ holds at all times.

\subsection{Target observables}

Using the methodology outlined above, we will present the results of some toy models (described in Section 3.2) for the following observables:

(i) the evolution of the galaxy size distribution of MGs [i.e. the size function $\left.\phi\left(R_{\mathrm{e}}, z \mid M_{\mathrm{star}}>10^{11.2} \mathrm{M}_{\odot}\right)\right]$ and its integral, the number density of MGs

$n_{\mathrm{MGs}}(z)=\int_{-\infty}^{\infty} \phi\left(R_{\mathrm{e}}, z \mid M_{\mathrm{star}}>10^{11.2} \mathrm{M}_{\odot}\right) \mathrm{d} \log R_{\mathrm{e}} ;$

(ii) the mean size of the population of MGs as a function of redshift, $\left\langle R_{\mathrm{e}}(z)\right\rangle$;

(iii) the evolution in the number density of compact MGs $n_{\text {compact }}(z)$. A range of definitions of compactness have been proposed 
in the literature (e.g. Saracco et al. 2010; Barro et al. 2013, 2017; Carollo et al. 2013; Fang et al. 2013; van der Wel et al. 2014; Damjanov et al. 2015; van Dokkum et al. 2015; Charbonnier et al. 2017; Tacchella et al. 2017; Buitrago et al. 2018; Tortora et al. 2018; Luo et al. 2020 among many others). Here, we define galaxies as compact systems if their size is 0.4 dex below the $z \sim 0 R_{\mathrm{e}}-M_{\text {star }}$ relation of quenched galaxies (Cassata et al. 2011, 2013),

$n_{\text {compact }}(z)=\int_{-\infty}^{-0.4} \phi\left(R_{\mathrm{e}} / R_{\mathrm{e}}(z=0), z\right) \mathrm{d} \log \left(R_{\mathrm{e}} / R_{\mathrm{e}}(z=0)\right)$.

In particular, we focus on compact quenched MGs (CQMGs) and compact star-forming MGs (CSFMGs).

Fig. C1 shows that adopting other definitions of compactness based on the effective radius yields qualitatively similar results to the Cassata et al. (2011) definition. Other popular definitions of compactness based on, e.g. the stellar mass density in the central kiloparsec, would require information on the light/mass profile of galaxies (e.g. the Sérsic index), which we are not including here. This requires further modelling which we defer to future work.

\section{RESULTS}

\subsection{At the core of the model}

The methodology outlined in Section 2 makes use of only two ingredients: (i) the K13 relation (equation 4) and (ii) the SMHM relation (most notably the high-mass slope $\delta$ and the scatter $\sigma_{\text {SMHM }}$ ).

Fig. 1 shows that two SMHM relation with different high-mass slope $\delta$ and scatter $\sigma_{\text {SMHM }}$ are able to produce the same number density for MGs. The degeneracy between $\delta$ and $\sigma_{\text {SMHM }}$ in producing the same abundances of MGs was already identified in previous studies (e.g. Behroozi, Conroy \& Wechsler 2010; Shankar et al. 2014). What we emphasize here, for the first time to the best of our knowledge, is that the corresponding halo mass distributions (middle panel, see also Shankar et al. 2014), and thus the implied size functions computed via the linear $R_{\mathrm{e}}-R_{\mathrm{h}}$ relation (right-hand panel), remain, however, significantly distinct, especially below the peaks of the distributions. The larger abundances of compact MGs are mostly driven by a larger scatter in the input SMHM relation, as can be inferred by comparing black dot-dashed and grey dashed lines in Fig. 1. Thus, the abundance of compact galaxies represents a valuable observable to break the degeneracy between $\delta$ and $\sigma_{\text {SMHM }}$, allowing to set constraints on the degree of progenitor bias and ultimately to discriminate between different models of galaxy formation.

\subsection{Toy models}

Motivated by the discussion above, we devise four toy models to show the effect of varying $\delta$ and $\sigma_{\text {SMHM }}$ on our target observables (Section 2.3):

(i) Model 1: $\delta=0.5$ (steep slope), $\sigma_{\mathrm{SMHM}}=0.15 \mathrm{dex}$ at all redshifts;

(ii) Model 2: $\delta=0.5$ (steep slope), $\sigma_{\mathrm{SMHM}}=\sqrt{(0.1 z)^{2}+0.15^{2}}$;

(iii) Model 3: $\delta=0.35$ (shallow slope), $\sigma_{\mathrm{SMHM}}=0.15 \mathrm{dex}$ at all redshifts;

(iv) Model 4: $\delta=0.35$ (shallow slope), $\sigma_{\mathrm{SMHM}}=$ $\sqrt{(0.1 z)^{2}+0.15^{2}}$.

The slope of Model 1 (Model 2) is inspired to the Grylls et al. (2020a) 'PyMorph' ('cmodel') SMHM relation, which was obtained by fitting the Bernardi et al. (2017) 'PyMorph' ('cmodel') SMF at $z \sim 0.1$ and the Davidzon et al. (2017) SMFs at $z \gtrsim 0.2$ where their masses have been corrected by 0.15 dex to bring the two studies in agreement $^{5}$ (see also Bernardi et al. 2016). ${ }^{6}$

Although some authors point to distinct SMHM relations for quiescent and star-forming galaxies (e.g. Rodríguez-Puebla et al. 2015; Moster et al. 2018; Behroozi et al. 2019; Posti \& Fall 2021), the relative content of stars in star-forming and quiescent galaxies at fixed halo mass is still highly debated (e.g. Wechsler \& Tinker 2018). We here adopt throughout the simplest assumption that quiescent and star-forming galaxies share the same underlying SMHM relation, and note that of our core results do not qualitatively depend on this working assumption.

\subsection{Halo occupation and implied size function}

As a first step, in Fig. 2 we show the SMHM relation and its scatter for the four toy models, as well as the distribution of the host haloes (i.e. the halo occupation distribution) and the implied size functions. Fig. 2 reveals that different SMHM relations and their scatter $\sigma_{\text {SMHM }}$ provide significantly different size functions, which necessarily stem from distinct host halo occupation distributions. Thus, the size functions are completely regulated by the way the SMHM relation maps galaxies into haloes. In particular, it is relevant to highlight the following features when comparing different models for the input SMHM relation:

(i) Model 1 versus Model 3. A change in the high-mass slope of the SMHM relation generates an overall lower number density of MGs, but the mean of the halo occupation distributions and related size functions are fairly similar in the two cases.

(ii) Model 1 versus Model 2 and Model 3 versus Model 4. Even when the shape of the SMHM relation is identical, if we allow for the scatter $\sigma_{\text {SMHM }}$ to evolve with redshift, and in particular to increase at earlier epochs, then the implied halo occupation distribution drastically changes compared to the case with constant scatter. In the former case, a higher proportion of small MGs are hosted in less massive haloes at higher redshift, and the mean halo occupation and galaxy size exhibit a stronger evolution, as quantitatively described below.

\subsection{Implied size evolution}

S14 showed that, on the assumption that $R_{\mathrm{e}} \propto R_{\mathrm{h}}$ at all epochs, the progressive increase in virial radii and in the number densities of massive dark matter haloes, were sufficient conditions to produce, when averaging over the full population, a strong size evolution in the sizes of MGs.

Fig. 3 confirms and further extends the claim by S14. By using, for each of our four toy models, a constant proportionality $R_{\mathrm{e}}=$ $A_{K} \times R_{\mathrm{h}}$ calibrated at $z=0.1$ (see Appendix B), as labelled, we are always able to reproduce the strong redshift evolution seen in the available data (Faisst et al. 2017; Patel et al. 2017; Mowla et al. $2019 b$ ), irrespective of the exact input SMHM relation. Models with an evolving $\sigma_{\text {SMHM }}$ tend to predict up to less than 50 per cent faster size evolutions, well within the variance currently found in the data. We distinguish between star-forming and quiescent galaxies via the $f_{\text {Quench }}$ model with $\mu=2$. Varying the $\mu$ parameter has little effect on our results, as it can be easily compensated by a relative variation

\footnotetext{
${ }^{5}$ This was done only for the 'PyMorph' SMF. The factor of 0.15 dex takes into account the difference in $M / L$ used in the two studies.

${ }^{6}$ We also shift by -0.1 dex the knee of the SMF resulting from the Grylls et al. (2020a) SMHM to better match the SDSS SMF.
} 

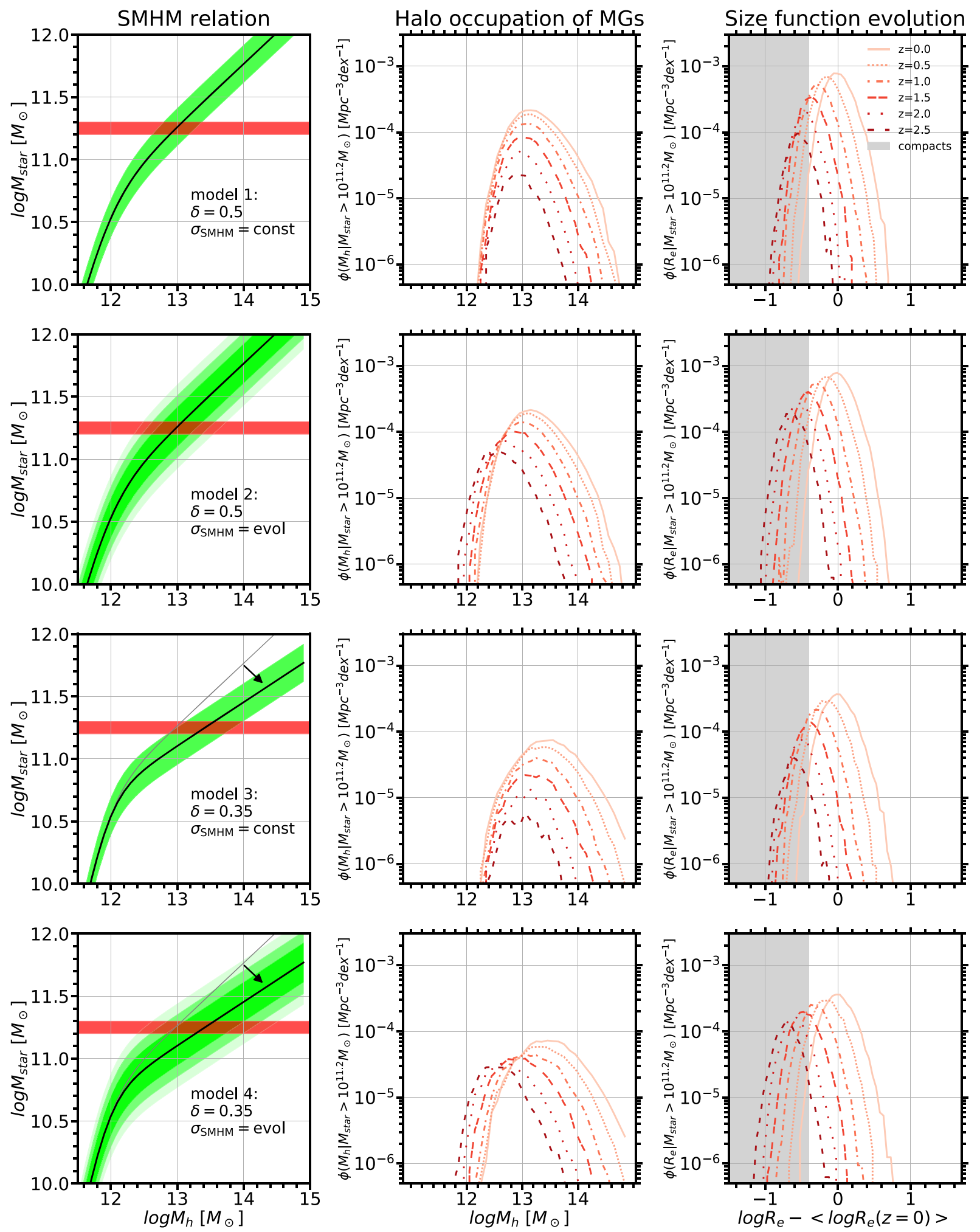

Figure 2. Left column: The SMHM relation of the four models outlined at the beginning of Section 3.2. The red line indicates the stellar mass selection for MGs. The green shaded regions indicate the scatter of the SMHM, which increases at higher redshift for Models 2 and 4 . The grey line in the centre-bottom and bottom panels indicates the SMHM for Models 1 and 2 as a reference. Central and Right column: The redshift evolution of the HOD $\phi\left(M_{\mathrm{h}} \mid M_{\mathrm{star}}>10^{11.2} \mathrm{M}_{\odot}\right)$ and the implied size functions $\phi\left(R_{\mathrm{e}} \mid M_{\mathrm{star}}>10^{11.2} \mathrm{M}_{\odot}\right)$ of MGs for the four models. We display results for $z=0,0.5,1,1.5,2,2.5,3$. Darker colours indicate higher redshift. The grey band in the right column shows the Cassata et al. (2013) definition for compact galaxies. It can be seen that the increasing $\sigma_{\mathrm{SMHM}}$ of Models 2 and 4 results in broader distributions, which have a median lower $M_{\mathrm{h}}$ and normalized $R_{\mathrm{e}}$ compared to Models 1 and 3 , where $\sigma_{\mathrm{SMHM}}=0.15$ dex at all times. An evolving $\sigma_{\mathrm{SMHM}}$ also results in a higher number density of MGs at earlier times. Contrariwise, the flatter high-mass-end slope of the SMHM in Model 3 results in overall fewer MGs and slightly larger median halo masses compared to Model 1. 

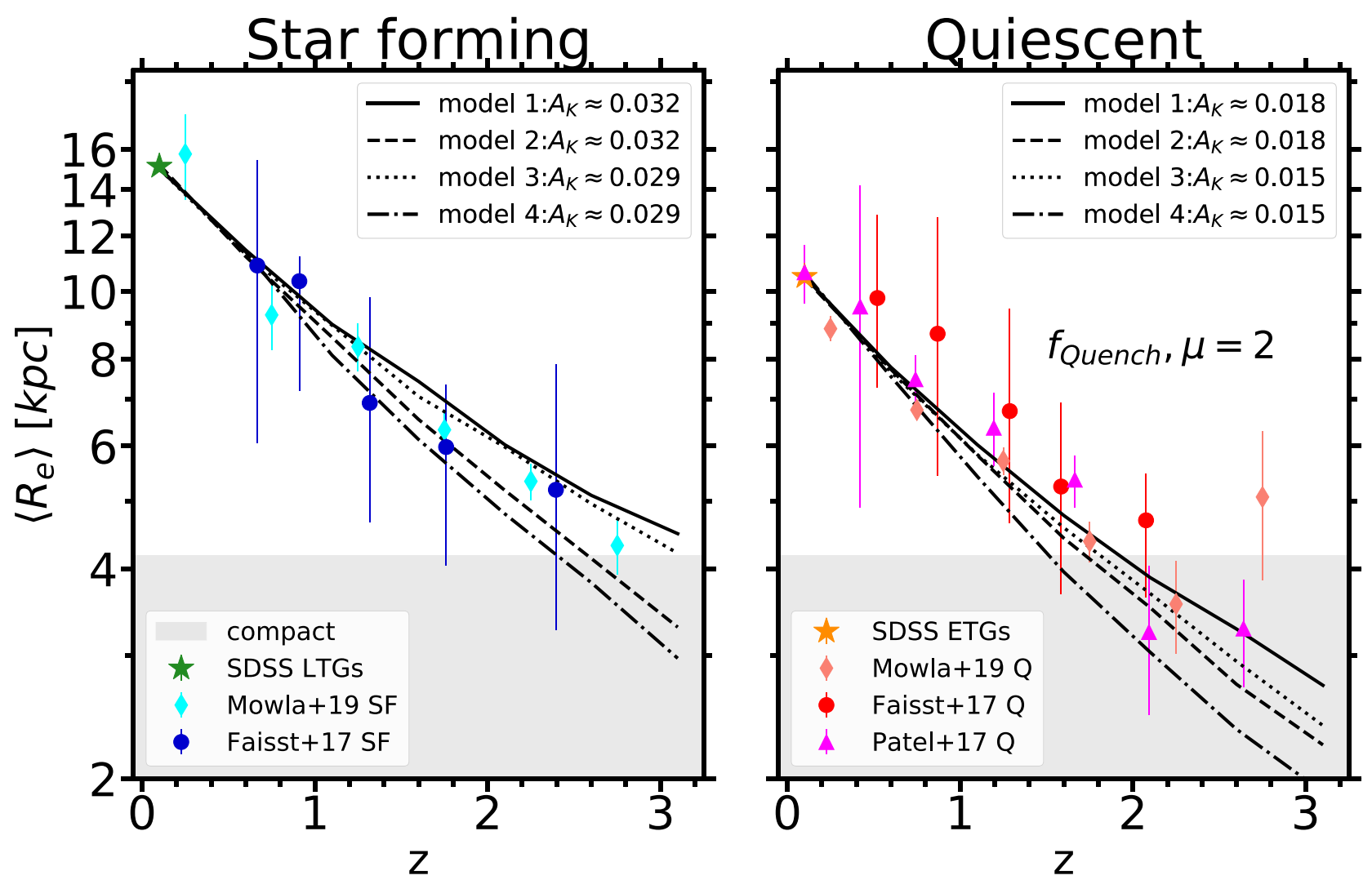

Figure 3. The size evolution from the K13 model for star-forming (left) and quiescent (right) MGs. The black lines indicate the four toy models outlined at the beginning of Section 3.2 and coupled with the $f_{\text {Quench }}$ model introduced in Section 2.2 with $\mu=3$, as an example. Data are the sizes of MGs from Mowla et al. (2019b) (diamonds), Faisst et al. (2017) (circles), Patel et al. (2017) (triangles). We also add SDSS estimates for the sizes of Massive Late type and Early type galaxies (green and orange stars, respectively) from Z20. The normalization $A_{K}$ in each panel is chosen to match SDSS observations. Notably, a constant normalization $A_{K}$ is able to reproduce observations. Moreover, $A_{K}$ is lower for shallower high-mass-end slopes of the SMHM (Models 1 and 2), while the opposite is true for steeper SMHM relations. This indicates that $A_{K}$ and $\delta$ are degenerate in our model.

in $A_{K}$ and/or in the SMHM relation. Indeed, the $A_{K}$ retrieved for starforming and quenched MGs appear to be systematically different and such difference persists even when adopting distinct SMHM relations as, for example, in Moster et al. (2018), for which we find $A_{K, S F} \approx 0.023$ and $A_{K, Q} \approx 0.016$.

\subsection{Implied statistics of compact MGs}

In Fig. 2, we showed that the shape and scatter of the SMHM have a significant impact on the number density of compact galaxies, a feature that was not investigated by previous studies. We explore these trends more quantitatively here for our toy models. The top and bottom panels of Fig. 4 show the predictions of Model 1 (constant scatter) and Model 2 (evolving scatter) for the number density of MGs (left-hand panels) and for only compact MGs (right-hand panels), separately for quiescent (red) and star-forming (cyan) galaxies and for different values of the quenching parameter $\mu$, as labelled (the predictions for Models 2 and 3 are very similar and reported in Appendix D). All models predict a similarly sharp rise in the number density of compact quiescent MGs (red lines) up to $z \sim 1.5-2$ and a subsequent more or less fast drop depending on the exact value of $\mu$ adopted. All models also predict the abundances of star-forming compact MGs (cyan lines) to peak around the same redshift $z \sim 2.5$ with a weak dependence on $\mu$ but a strong one on scatter: a larger $\sigma_{\text {SMHM }}$ at early epochs can increase by up to a factor of 10 the predicted number densities of star-forming compact MGs (bottom right panel). In Appendix C, we show that adopting other definitions of compactness (e.g. Barro et al. 2013; van der Wel et al. 2014; Gargiulo et al. 2017) does not alter the main qualitative trends of Fig. 4.

The evolution of $n_{\text {compact }}$ that we predict for compact quiescent MGs is in qualitative agreement with observations of compact galaxies in a lower mass range $\left(10.5<\log M_{\text {star }} \mathrm{M}_{\odot}<11.5\right.$; Cassata et al. 2011, 2013; Barro et al. 2013; van der Wel et al. 2014). However, at present, current observations provide rather uncertain constraints on $n_{\text {MGs }}$ at high redshift (see Kawinwanichakij et al. 2020 for a detailed discussion of the systematics). In addition, a secure determination of the number density of, especially compact, MGs is hampered by the seizable but still unknown number of optically dark star-forming galaxies at high redshift (e.g. Franco et al. 2018; Wang et al. 2019; Zhou et al. 2020; Smail et al. 2021). Nevertheless, the results presented in Fig. 4 provide clear predictive trends for the evolution of compact and large MGs that, when compared with data from the next generation of observing facilities, will set tight constraints on the quenching mechanisms ( $\mu$ parameter) and on the level of progenitor bias in the size evolution of MGs.

\section{DISCUSSION}

\subsection{Progenitor bias scenarios and continuity equation}

We have demonstrated that all our models are able to produce a strong evolution in the average effective radius of the MG populations 

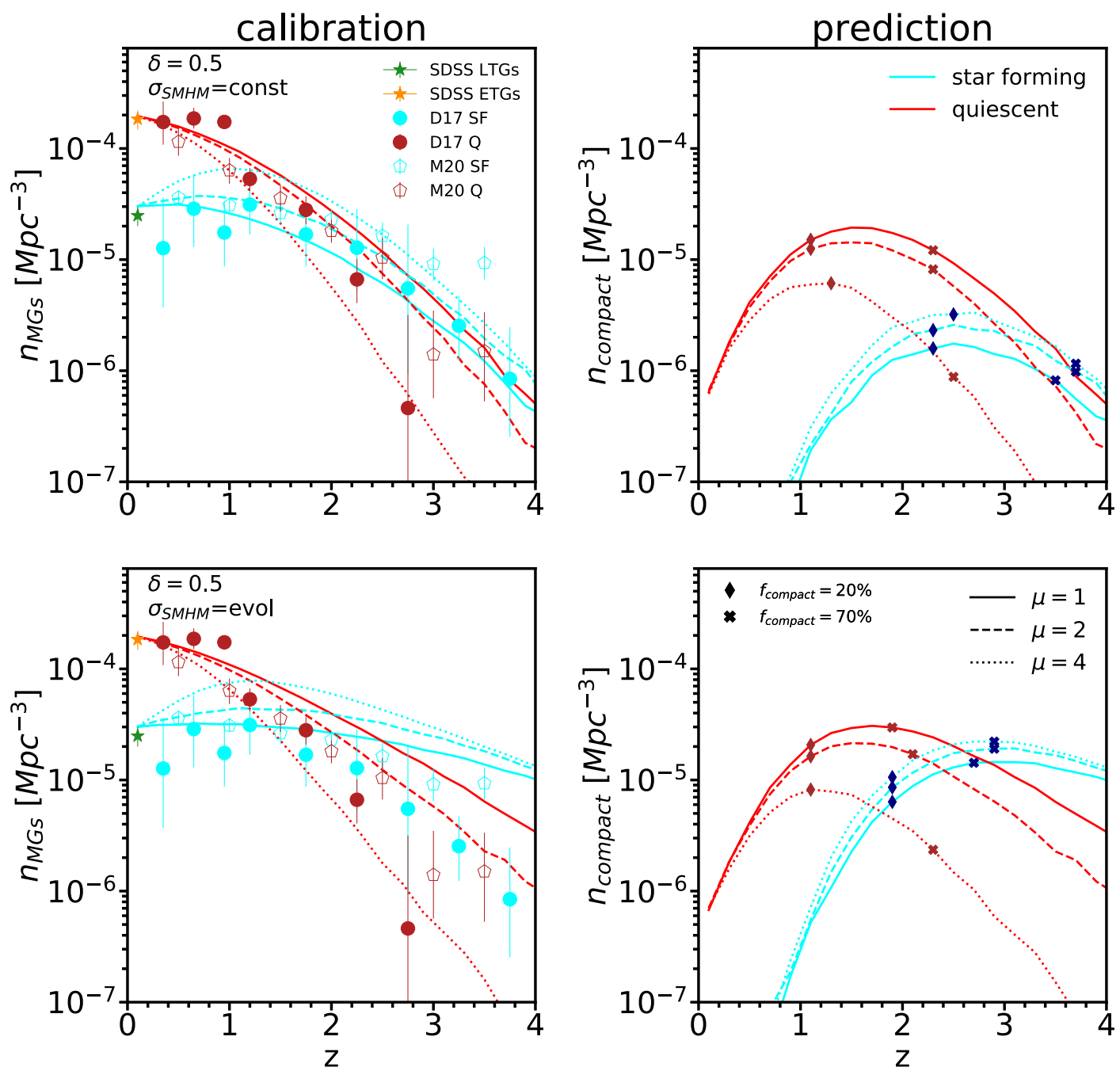

Figure 4. Left: The number density of star-forming and quenched MGs (cyan and red lines, respectively) for models 1 and 2. Solid, dashed, and dotted lines are obtained adopting $\mu=1,2$, and 4, respectively. Right: Prediction for the number density of compact MGs for the two models and the different values of $\mu$. Note that the fraction of compact MGs increases at early times. Filled diamonds and crosses indicate the time where 20 per cent and 70 per cent of the population of MGs (either star-forming or quenched) are compact. The comparison data in the left column are from the SDSS 'PyMorph' photometry at $z=0.1$ (Meert, Vikram \& Bernardi 2015), Davidzon et al. (2017) and McLeod et al. (2021) (in the wo latter cases the masses have been shifted by 0.15 dex to account for M/L differences with our SDSS data, see Grylls et al. 2020a). Note that the data points were retrieved from the Schechter fits provided in the two studies, extrapolated in our mass range. With the caveat that different definitions of quiescence are adopted in observations, we note that Model 1 is favoured by current data if $\mu \approx 2-3$. Model 2 might provide a better fit to data if the number density of star-forming MGs is underestimated at high redshift (Franco et al. 2018; Smail et al. 2021).

(Fig. 3). On the other hand, Figs 4 and D1 clearly show that in all models $n_{\text {compact }}$ decreases below $z \sim 1.5-2$. The peak of the abundance of CQMGs corresponds to compact fractions of $\sim 20$ 40 per cent. Thus, the ensuing disappearance of compact galaxies as the universe ages strongly suggests that $\sim 20-40$ per cent of the quenched MGs that were present at $z \sim 1.5-2$ have grown in size individually (e.g. Trujillo, Ferreras \& de La Rosa 2011; Carollo et al. 2013; van der Wel et al. 2014; Fagioli et al. 2016; Faisst et al. 2017; Stockmann et al. 2021). However, it is worth pointing out that this corresponds to only $\sim 10-15$ per cent of the quenched MGs that are present today (for the case of constant and evolving $\sigma_{\mathrm{SMHM}}$, respectively).

At $z \gtrsim 2$ all models instead predict a strong increase in the number density of compact MGs, suggesting that, in line with a number of observational studies (e.g. Barro et al. 2013; Cassata et al. 2013), a significant fraction of MGs form in a compact phase at early epochs, most probably due to gas dissipation following a merger (e.g. Sparre \& Springel 2016) or an in situ burst of star formation (e.g. Lapi et al. 2011).

An interesting question that has been discussed in the literature is whether compact quenched galaxies are the descendants of compact star-forming galaxies (e.g. van Dokkum et al. 2015; Barro et al. 2017; Gómez-Guijarro et al. 2019). For example, based on number density conservation arguments, Barro et al. (2013) proposed that compact star-forming galaxies with $10.5<\log M_{\text {star }} \mathrm{M}_{\odot}<11.5$, passively evolve into quenched compact galaxies in a time-scale of $\sim 800$ Myr. Following Barro et al. (2013), we here develop basic continuity equation models without mergers in which CSFMGs naturally evolve into CQMGs on a given time-scale $\Delta T_{\text {quench }}$ as

$n_{\mathrm{CSFMGs}}(t)=n_{\mathrm{CQMGs}}\left(t+\Delta T_{\text {quench }}\right)-n_{\mathrm{CQMGs}}(t)$ 

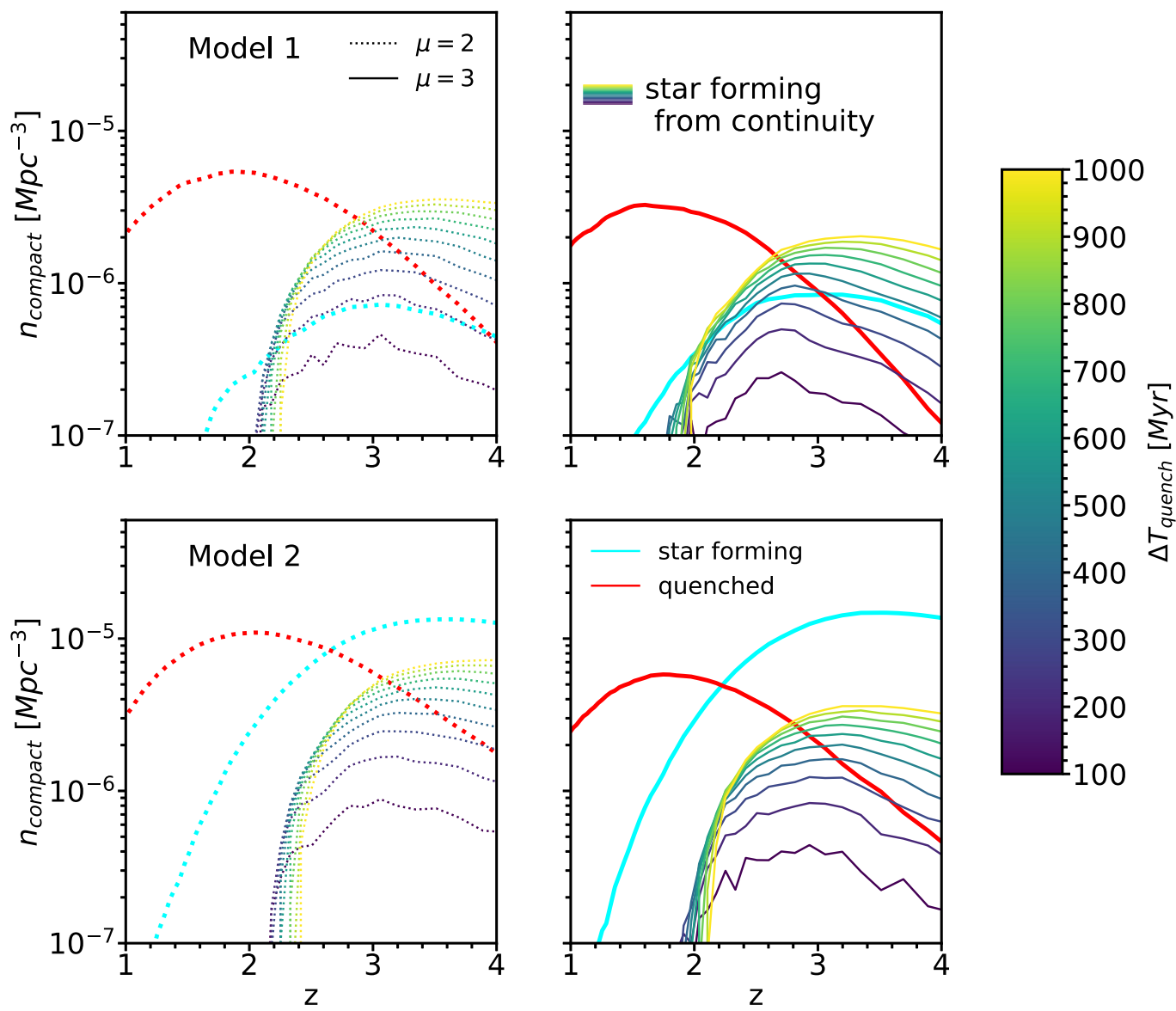

Figure 5. The number density of CSFMGs (cyan) and CQMGs (red) for Model 1 (top row) and Model 2 (bottom row). We adopt mu $\mu=2$ (dotted lines, left columns) and $\mu=3$ (solid lines, right column). The number density of compact star-forming galaxies that would be obtained from continuity arguments (see

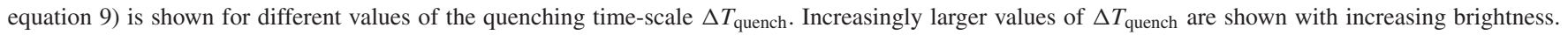
Model 2 disfavours a continuity scenario. In Model 1 continuity is broadly achieved if $\Delta T_{\text {quench }} \approx 200-400$ Myr for $\mu=2$ and $\mu=3$, respectively. Results for Models 3 and 4 can also be found in the online supporting material, and are qualitatively similar.

in which $\Delta T_{\text {quench }}$ is allowed to vary between 100 and $900 \mathrm{Myr}, t$ is the age of the Universe, and $n_{\mathrm{CQMGs}}(t)$ and $n_{\mathrm{CSFMGs}}(t)$ are the cumulative number densities of quiescent and star-forming compact MGs above $M_{\text {star }}>10^{11.2} \mathrm{M}_{\odot}$. Fig. 5 shows the results of applying equation (9) to the $n_{\mathrm{CSFMGs}}$ extracted from Models 1 and 2 (see online supporting material for Models 3 and 4) with quenching parameters $\mu=2,3$ as a reference (the results derived for other values of $\mu$ are included in the online supporting material).

As reported in the bottom panel of Fig. 5, models characterized by a scatter $\sigma_{\mathrm{SMHM}}$ increasing at earlier epochs tend to disfavour a continuity scenario in which all CSFMGs gradually transition into CQMGs, as the number densities of CSFMGs (cyan lines) are always significantly larger than those of compact quiescent galaxies (red lines). Instead, models with a fixed $\sigma_{\text {SMHM }}$ (Model 1 , top row of Fig. 5) are broadly consistent with a progenitor-descendant scenario between CSFMGs and CQMGs for some choices of $\Delta T_{\text {quench. In the }}$ specific, we find that $\Delta T_{\text {quench }} \approx 200,300,400,900$ Myr for $\mu=$ $2,2.5,3,4$ (data shown only for $\mu=2,3$, see online supporting material for $\mu=2.5,4)$. Thomas et al. (2005) estimated an upper limit to the main star formation episode of local MGs around $\Delta T_{\text {quench }}$ $\lesssim 300 \mathrm{Myr}$ (see their equation 5), which would be consistent, at face value, with continuity in our constant $\sigma_{\text {SMHM }}$ models with $2 \lesssim \mu \lesssim$ 3 , in line with our preferred values of $\mu$ adopted in Figs 4 and D1.
We note that continuity arguments applied to Models 3 and 4 (see online supporting material) yield results that are qualitatively similar to Models 1 and 2, respectively.

In a continuity scenario between CSFMGs and CQMGs (which can be produced by Models 1 and 3), little or no size evolution occurs during quenching. This conflicts with theoretical models where both size evolution and quenching occur almost simultaneously as a result of AGN activity, with a predicted expansion in size of a factor of $\gtrsim 2$ over very short time-scales (i.e. 50-100 Myr; Ragone-Figueroa \& Granato 2011; Lapi et al. 2018a). In other words, assuming a constant scatter $\sigma_{\text {SMHM }}$ in the input SMHM relation, would be consistent with a two-stage formation scenario in which galaxies first quench and then grow via stochastic mergers (e.g. Hopkins et al. 2009; Oser et al. 2012). Alternatively, an increasing $\sigma_{\text {SMHM }}$ at earlier epochs would necessarily require within our framework that only a relatively minor fraction of the CSFMGs quench during their compact phase, a scenario more consistent with an AGN-driven size evolution. We note that an unbiased view of the size growth of MGs requires both optical-NIR observations as well as FIR-submm observations (e.g. Barro et al. 2016; Tadaki et al. 2020; Sun et al. 2021). Compact dust-enshrouded star formation activity can in fact occur over spatial scales a factor of $\sim 3$ smaller that the $R_{e}$ measured in optical-NIR (e.g. Jiménez-Andrade et al. 2019; Puglisi et al. 2019). 
AGN activity in these galaxies might cause, along with quenching, a considerable evolution in size in a very short time-scale (e.g. Lapi et al. 2018a).

We conclude this section by stressing the fact that our continuity models strictly apply to compact MGs, which amount to a substantial fraction of the total population of quiescent MGs only at $z \gtrsim 2$ (see diamonds and crosses on Fig. 4). It is evident from Fig. 4, that all models predict an increase in the number density of the overall quiescent population at $z<2$ by up to an order of magnitude, a trend that cannot be driven by solely quenching of the star-forming MGs as the number density of the latter is always significantly lower than those of quenched MGs at late epochs. Additional physical processes must be at play at $z<2$ in regulating the formation and sustenance of non-compact star-forming MGs as well as the appearance of a large population of non-compact quenched MGs.

\subsection{The sizes of MGs as effective constraints to the galaxy-halo connection}

Providing firm constraints to the SMHM relation at different epochs can yield invaluable information on, e.g. the merger rates of MGs (Grylls et al. 2020b), the interplay between dark matter and baryonic physics (Gu et al. 2016; Matthee et al. 2017), the physical processes behind galaxy quenching (Tinker 2017). Unfortunately, the shape and scatter of the SMHM relation are still highly debated (e.g. Bernardi et al. 2017). In particular, there is a well-known degeneracy between the high-mass slope, $\delta$, and the dispersion, $\sigma_{\text {SMHM }}$, of the SMHM relation (e.g. Shankar et al. 2014). Similarly to Grylls et al. (2020b), in the previous sections we made use of toy models where only these two parameters are changed to explore their impact on the sizes of MGs. As shown above, SMHM relations with different values of $\delta$ and $\sigma_{\text {SMHM }}$ result in distinct rates of size increase (Fig. 3) and number density evolution of compact MGs (Figs 4 and D1), which are ultimately a consequence of the different implied HOD (Fig. 1). Our results therefore suggest that the $\delta-\sigma_{\text {SMHM }}$ degeneracy may be broken by simultaneously fitting the size growth of MGs, the redshift evolution of the number density of compact MGs, and the number density evolution of the overall population of MGs, in other words by an accurate measurement of the full size function of MGs at different epochs, a goal that should be achievable with the aid of the next-generation observational facilities such as Euclid and LSST. We note that several previous semi-empirical studies aimed at probing the size evolution of galaxies (e.g. Rodríguez-Puebla et al. 2017; Hearin et al. 2019; Behroozi, Hearin \& Moster 2021). However, they were are all limited by the use of only one SMHM relation and dispersion, which instead, if allowed to vary, can provide distinct structural evolutionary tracks for MGs.

It is important to highlight that the systematic uncertainties in measuring stellar masses and number densities of galaxies substantially affect the determination of the SMF, and thus of the SMHM relation and size distributions of MGs at different epochs, possibly explaining at least part of the observational discrepancies in the numbers of compact galaxies reported in the literature (e.g. Poggianti et al. 2013).

\subsection{Covariance between the $\boldsymbol{R}_{\mathrm{e}}-\boldsymbol{R}_{\mathrm{h}}$ and the SMHM relations?}

A further interesting issue that warrants more exploration is that of a possible covariance between the $R_{\mathrm{e}}-R_{\mathrm{h}}$ and the SMHM relation. In our framework, the $R_{\mathrm{e}}-R_{\mathrm{h}}$ and the SMHM relations are closely intertwined. However, we did not consider an explicit correlation between the two relations which, instead, may be possible. For example, SDSS observations (Bernardi et al. 2014) have shown that at fixed velocity dispersion (which is a proxy of halo mass, e.g. Sohn et al. 2020) brighter (i.e. more massive) galaxies have larger $R_{\mathrm{e}}$ (Shankar \& Bernardi 2009). Such a trend may be captured by introducing a positive covariance between the scatters of the SMHM and of the K13 relation. We ran a few simple tests and found that adding this ingredient to our framework does not significantly affect the implied size evolution of MGs. When including a positive covariance, less MGs tend to also naturally be the smallest galaxies. The covariance thus ultimately generates narrower size function at fixed stellar mass, where the abundance of compact galaxies is now only controlled by the dispersion in size at fixed halo radius $\sigma_{K}$. Therefore, a higher $\sigma_{K}$, we find, can produce the same amount of compact galaxies as in a model without covariance but with a proportionally lower value of $\sigma_{K}$. The degeneracy between $\sigma_{K}$ and a covariance between the SMHM and the K13 relations may be broken by probing the environmental dependence of galaxy size at fixed stellar mass, a task that is beyond the scope of this work.

\subsection{Including concentration in the K13 model}

Some authors have argued that galaxy sizes may be regulated also by halo concentration (Desmond 2017; Desmond et al. 2019; Jiang et al. 2019). Essentially, this 'concentration model' is a modified version of the K13 model where an inverse proportionality between galaxy size and halo concentration is also considered,

$$
\begin{aligned}
R_{\mathrm{e}} & =A_{c}\left(\frac{c}{10}\right)^{\gamma} R_{\mathrm{h}} \\
& =f(c) R_{\mathrm{h}},
\end{aligned}
$$

where we define $f(c)=A_{c}\left(\frac{c}{10}\right)^{\gamma}$ and $\gamma<0$. Here, we will leave $\gamma$ as a redshift-independent free parameter. For the concentration, we adopt the concentration-mass relation by Dutton \& Macciò (2014),

$\log c=a+b \log M_{\mathrm{h}}[\mathrm{M} \odot] / 10^{12} / h$

with $a(z)=0.537+(1.025-0.537) \exp \left(-0.718 z^{1.08}\right)$ and $b(z)=$ $-0.097+0.024 z$. Dutton \& Macciò (2014) report a lognormal scatter of about $\sim 0.11$ dex which is independent on halo mass.

The results from the concentration model with $\gamma=-0.4,-0.6$, -0.8 are reported in Fig. 6 for the four toy models explored in this paper (see Section 3.2). The most important feature of this figure is that all models struggle to reproduce the size evolution of MGs, except for Model 4 characterized by a flat high-mass SMHM slope $\delta$ and an evolving scatter $\sigma_{\text {SMHM }}$. All models predict an increase in size at fixed stellar mass, with higher (absolute) values of $\gamma$ generating a shallower evolution. As $\gamma$ approaches zero, the trend tends to reduce to that of the K13 model, as expected. The departure from the K13 model is explained by the evolution of the factor $f(c)=(c / 10)^{\gamma}$ (equation 10, Fig. 7), which has the effect of slowing down the evolution with respect to the K13 model. The predicted relatively slower size evolution in the concentration model is roughly independent of the input SMHM due to the shallow correlation between halo mass and concentration (see equation 11). Although the concentration model struggles to reproduce a strong size evolution, as already noted by Jiang et al. (2019), it cannot still be ruled out as current data may be underestimating galaxy sizes at high redshift due to surface brightness (e.g. Ribeiro et al. 2016; Whitney et al. 2019) and/or colour gradients effects (e.g. van der Wel et al. 2014; Mosleh et al. 2017; Suess et al. 2019, 2020). 
model 1

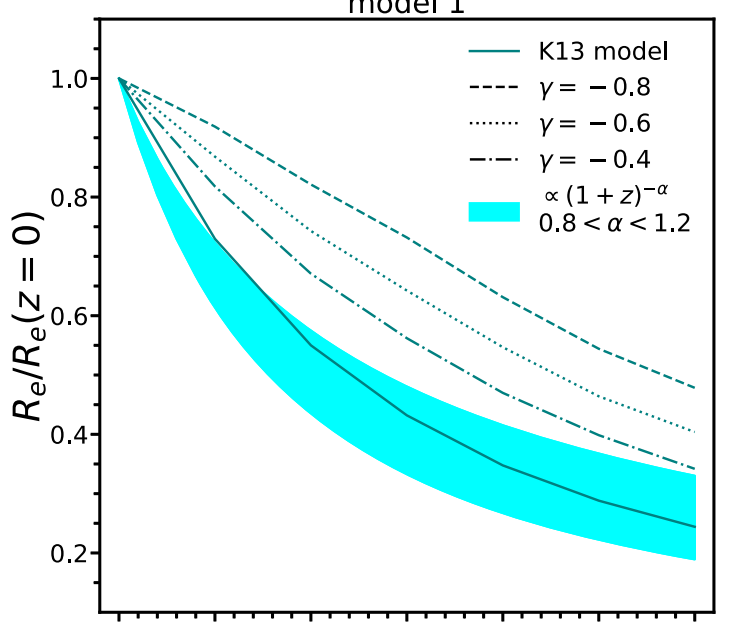

model 3

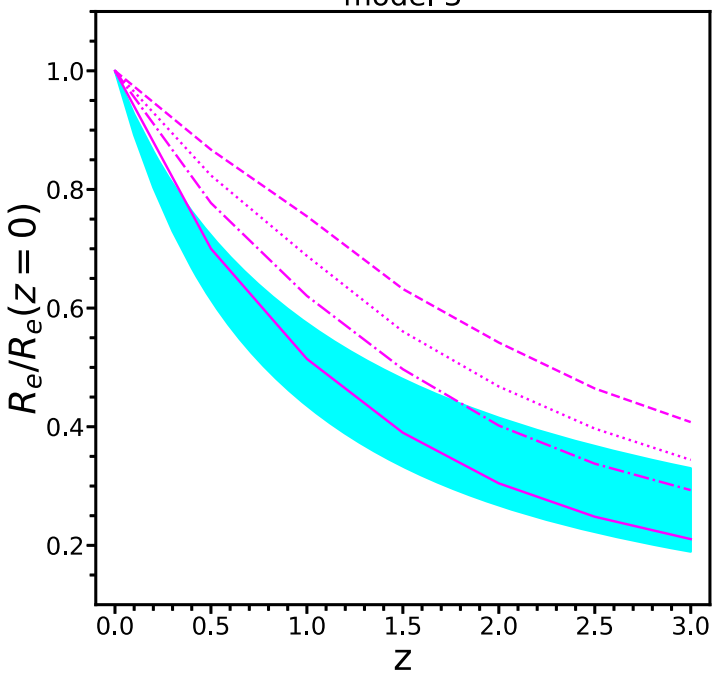

model 2

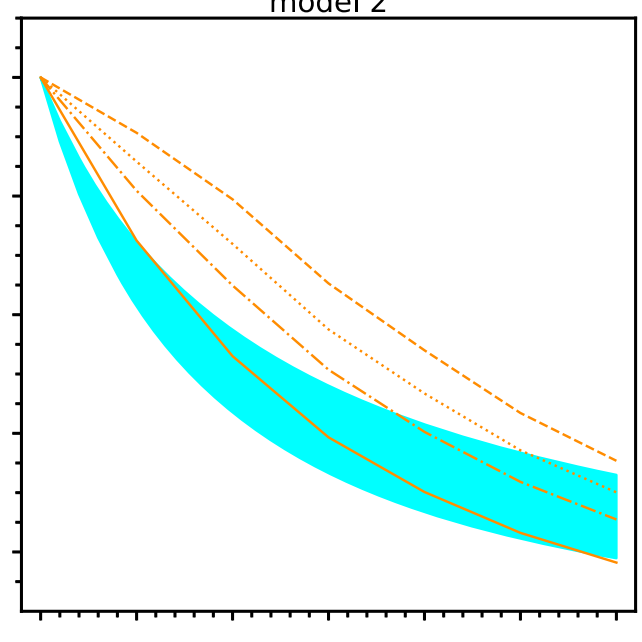

model 4

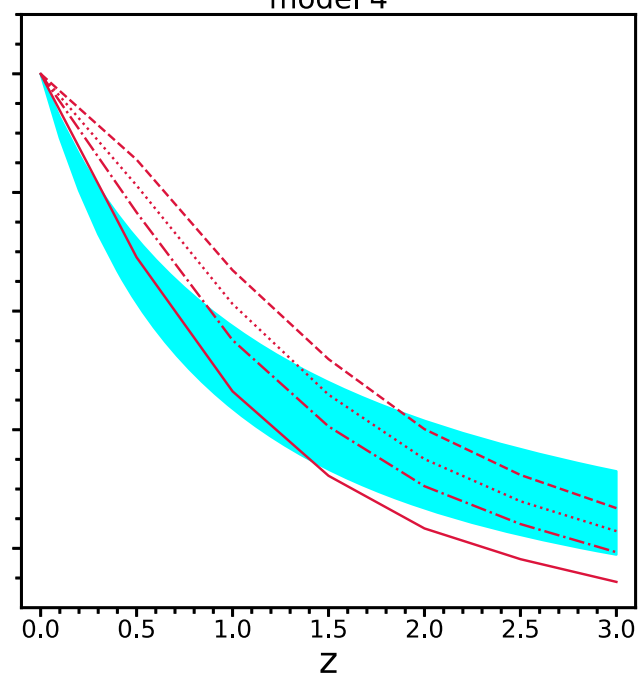

Figure 6. Size evolution inferred from the concentration model for $\gamma=-0.4,-0.6,-0.8$ (dot-dashed, dotted, and dashed lines, respectively). Top left is for Model 1, top right for Model 2, bottom left for Model 3, and bottom right for Model 4. In all panels, solid lines indicate the K13 model). The cyan shaded area broadly indicates the range of observational constraints allowed by current data $\left[R_{\mathrm{e}} \propto(1+z)^{-\alpha}\right.$ with $-1.2<\alpha<-0.8$, see Faisst et al. (2017), Patel et al. (2017), Mowla et al. (2019b)]. All models struggle to reproduce the observed size evolution. Model 4, which has a shallow high-mass slope in the SMHM and for which an evolving $\sigma_{\text {SMHM }}$ is implemented, provides a better match to the observed trend for some values of $\gamma$.

\section{CONCLUSIONS}

In this work, we developed accurate and transparent semi-empirical models to study the evolution of the size (effective radius $R_{\mathrm{e}}$ ) function of MGs $\left(M_{\text {star }}>10^{11.2} \mathrm{M}_{\odot}\right)$. We assumed an input SMHM relation to populate dark matter haloes with galaxies, and then assigned sizes to galaxies via an empirically motivated linear and tight relation between $R_{\mathrm{e}}$ and the host halo virial radius $R_{\mathrm{h}}$. We varied the input SMHM relation to reflect the still substantial systematic uncertainties in the SMF at both low and high redshift (see Footnote 1 in the Introduction). More specifically, we devised four toy models with different high-mass slopes and/or dispersions at fixed halo mass, $\sigma_{\mathrm{SMHM}}$, to probe their impact on the size function of MGs. In particular, we focused on the mean size growth and number density evolution since $z \sim 3$ of compact star-forming and quiescent MGs, distinguished in the mocks via a simple halo massdependent quenching model with only one parameter (RodríguezPuebla et al. 2015). Our main results can be summarized as follows: (i) The shape and evolution of the size function are completely determined by the HOD implied by each model. In particular, the number density of compact galaxies, $n_{\text {compact }}$, is a strong function of the scatter $\sigma_{\text {SMHM }}$ (Figs 1 and 2).

(ii) All models are able to broadly reproduce the fast size growth of star-forming and quiescent MGs by simply assuming a redshiftindependent $R_{\mathrm{e}}-R_{\mathrm{h}}$ relation with a different zero-point for the two populations (Fig. 3) and in ways largely independent of the shape of the input SMHM relation and of its scatter.

(iii) In all models, the number density of CSFMGs peaks at around $z \sim 2.5$ and sharply declines at later times, while the peak in the number density of compact quiescent MGs is always delayed by a characteristic time-scale which depends on the specific model (Fig. 4). Our findings thus suggest a size growth driven by newly formed MGs at $z \gtrsim 1.5-2$, e.g. 'progenitor bias', which plays a gradually lesser (but still important) role at $z \lesssim 1.5$.

(iv) In models in which the scatter $\sigma_{\text {SMHM }}$ is strictly constant in time, we find that our predictions are consistent with a two-phase 


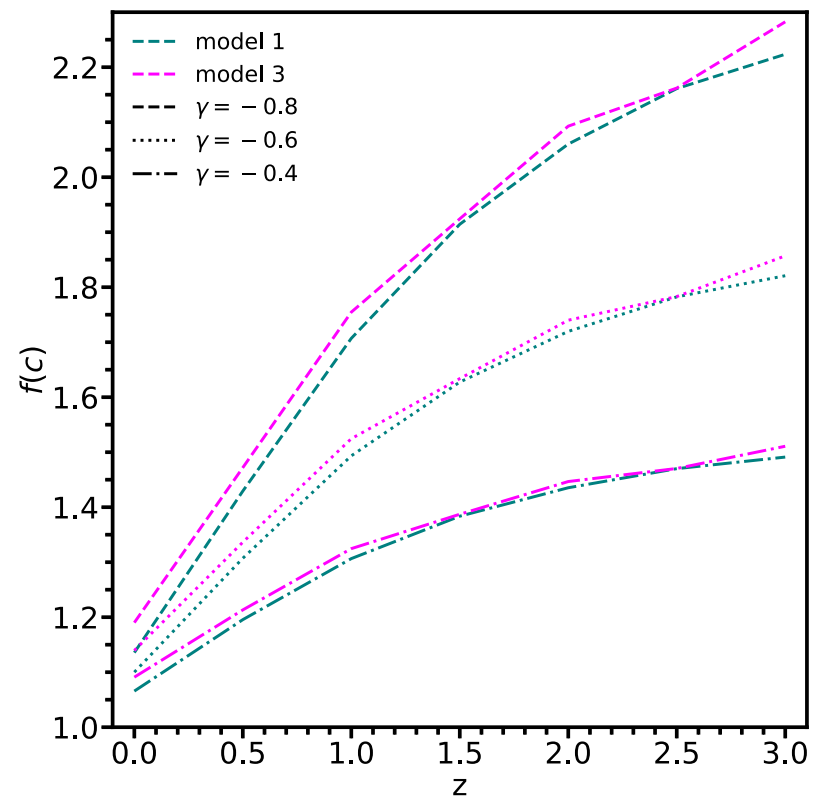

Figure 7. The redshift dependence of the factor $f(c)$ in the concentration model for $\gamma=-0.4,-0.6,-0.8$ (dot-dashed, dotted, and dashed lines, respectively), for Model 1 (teal lines) and Model 3 (magenta lines). $f(c)$ increases at earlier times, and it is weakly dependent on the SMHM relation.

evolution scenario, in which CSFMGs first quench into compact quiescent MGs on a time-scale of a few hundred Myr (Fig. 5), and then grow in size (possibly via dry mergers). In models in which $\sigma_{\text {SMHM }}$ is instead allowed to increase at earlier epochs, a significant proportion of quiescent MGs must increase their sizes before final quenching as in, e.g. AGN-driven size growth.

(v) We also implemented another variant of the models in which $R_{e}$ is proportional to virial radius via a halo concentration-dependent factor $f(c)$ (Jiang et al. 2019). We find that, at face value, this model struggles at reproducing the fast size growth of the population of MGs (Fig. 6), although the data may be underestimating galaxy sizes at high redshift.

All in all, our results support the view that an accurate measurement of the full size function of MGs, which will become available with the next generation of observing facilities such as EUCLID and the Nancy Grace Roman Space Telescope, will be able to set constraints on (i) the high-mass slope and scatter of the SMHM relation, (ii) the rate of evolution of the number density of compact quiescent and star-forming MGs and the related degree of progenitor bias, (iii) the quenching time-scales of star-forming MGs, and (iv) the evolutionary processes (mergers versus AGN feedback) driving the structural evolution of MGs.

\section{ACKNOWLEDGEMENTS}

We thank the anonymous referee for helping us improve the presentation of the results. LZ acknowledges funding from the Science and Technologies Facilities Council, which funded his PhD through the DISCnet Center for Doctoral Training. FS acknowledges partial support from a Leverhulme Trust Research Fellowship. We thank Ignacio Trujillo, Simona Mei, and Adriana Gargiulo for useful comments and Nushkia Chamba for providing data tables for the measurements of $R_{1}$. HF acknowledges support from the European Union's Horizon 2020 research and innovation programme under the Marie Skłodowska-Curie grant agreement No. 860744.

\section{DATA AVAILABILITY}

All data will be shared upon reasonable request to the authors. We provide our codes at https://github.com/lorenzozanisi/SizeModels.

\section{REFERENCES}

Abazajian K. N. et al., 2009, ApJS, 182, 543

Andreon S., 2013, A\&A, 554, A79

Barro G. et al., 2013, ApJ, 765, 104

Barro G. et al., 2016, ApJ, 827, L32

Barro G. et al., 2017, ApJ, 840, 47

Behroozi P. S., Conroy C., Wechsler R. H., 2010, ApJ, 717, 379

Behroozi P. S., Wechsler R. H., Conroy C., 2013, ApJ, 770, 57

Behroozi P., Wechsler R. H., Hearin A. P., Conroy C., 2019, MNRAS, 488, 3143

Behroozi P., Hearin A., Moster B. P., 2021, preprint (arXiv:2101.05280)

Bernardi M., Shankar F., Hyde J. B., Mei S., Marulli F., Sheth R. K., 2010, MNRAS, 404, 2087

Bernardi M., Roche N., Shankar F., Sheth R. K., 2011a, MNRAS, 412 , 684

Bernardi M., Roche N., Shankar F., Sheth R. K., 2011b, MNRAS, 412 , L6

Bernardi M., Meert A., Sheth R. K., Vikram V., Huertas-Company M., Mei S., Shankar F., 2013, MNRAS, 436, 697

Bernardi M., Meert A., Vikram V., Huertas-Company M., Mei S., Shankar F., Sheth R. K., 2014, MNRAS, 443, 874

Bernardi M., Meert A., Sheth R. K., Huertas-Company M., Maraston C., Shankar F., Vikram V., 2016, MNRAS, 455, 4122

Bernardi M., Meert A., Sheth R. K., Fischer J. L., Huertas-Company M., Maraston C., Shankar F., Vikram V., 2017, MNRAS, 467, 2217

Bryan G. L., Norman M. L., 1998, ApJ, 495, 80

Buitrago F., Trujillo I., Conselice C. J., Bouwens R. J., Dickinson M., Yan H., 2008, ApJ, 687, L61

Buitrago F. et al., 2018, A\&A, 619, A137

Cappellari M., 2016, ARA\&A, 54, 597

Carollo C. M. et al., 2013, ApJ, 773, 112

Cassata P. et al., 2011, ApJ, 743, 96

Cassata P. et al., 2013, ApJ, 775, 106

Chamba N., Trujillo I., Knapen J. H., 2020, A\&A, 633, L3

Charbonnier A. et al., 2017, MNRAS, 469, 4523

Chen Z. et al., 2020, ApJ, 897, 102

Cimatti A., Nipoti C., Cassata P., 2012, MNRAS, 422, L62

Conroy C., Wechsler R. H., 2009, ApJ, 696, 620

Daddi E. et al., 2005, ApJ, 626, 680

Damjanov I. et al., 2011, ApJ, 739, L44

Damjanov I., Geller M. J., Zahid H. J., Hwang H. S., 2015, ApJ, 806, 158

Davidzon I. et al., 2017, A\&A, 605, A70

De Lucia G., Blaizot J., 2007, MNRAS, 375, 2

Desmond H., 2017, MNRAS, 464, 4160

Desmond H., Mao Y.-Y., Wechsler R. H., Crain R. A., Schaye J., 2017, MNRAS, 471, L11

Desmond H., Katz H., Lelli F., McGaugh S., 2019, MNRAS, 484, 239

Despali G., Giocoli C., Angulo R. E., Tormen G., Sheth R. K., Baso G., Moscardini L., 2016, MNRAS, 456, 2486

Diemer B., 2018, ApJS, 239, 35

Domínguez Sánchez H., Huertas-Company M., Bernardi M., Tuccillo D., Fischer J. L., 2018, MNRAS, 476, 3661

Donnari M. et al., 2019, MNRAS, 485, 4817

Duncan K. et al., 2019, ApJ, 876, 110

Dutton A. A., Macciò A. V., 2014, MNRAS, 441, 3359

Dutton A. A., Conroy C., van den Bosch F. C., Prada F., More S., 2010, MNRAS, 407, 2

Erfanianfar G. et al., 2019, A\&A, 631, A175

Fagioli M., Carollo C. M., Renzini A., Lilly S. J., Onodera M., Tacchella S., 2016, ApJ, 831, 173 
Faisst A. L., Carollo C. M., Capak P. L., Tacchella S., Renzini A., Ilbert O., McCracken H. J., Scoville N. Z., 2017, ApJ, 839, 71

Fall S. M., 1983, in Athanassoula E., ed., Proc. IAU Symp 100, Internal Kinematics and Dynamics of Galaxies. Reidel, Dordrecht, p. 391

Fan L., Lapi A., De Zotti G., Danese L., 2008, ApJ, 689, L101

Fan L., Lapi A., Bressan A., Bernardi M., De Zotti G., Danese L., 2010, ApJ, 718,1460

Fang J. J., Faber S. M., Koo D. C., Dekel A., 2013, ApJ, 776, 63

Firmani C., Avila-Reese V., 2010, ApJ, 723, 755

Fischer J. L., Bernardi M., Meert A., 2017, MNRAS, 467, 490

Franco M. et al., 2018, A\&A, 620, A152

Gargiulo A., Saracco P., Tamburri S., Lonoce I., Ciocca F., 2016, A\&A, 592, A132

Gargiulo A. et al., 2017, A\&A, 606, A113

Gómez-Guijarro C. et al., 2019, ApJ, 886, 88

Grylls P. J., Shankar F., Zanisi L., Bernardi M., 2019, MNRAS, 483, 2506

Grylls P. J., Shankar F., Leja J., Menci N., Moster B., Behroozi P., Zanisi L., 2020a, MNRAS, 491, 634

Grylls P. J., Shankar F., Conselice C. J., 2020b, MNRAS, 499, 2265

Gu M., Conroy C., Behroozi P., 2016, ApJ, 833, 2

Guarnieri P. et al., 2019, MNRAS, 483, 3060

Guo Q. et al., 2011, MNRAS, 413, 101

Hearin A., Behroozi P., Kravtsov A., Moster B., 2019, MNRAS, 489, 1805

Hopkins P. F., Hernquist L., Cox T. J., Keres D., Wuyts S., 2009, ApJ, 691, 1424

Hopkins P. F. et al., 2010a, ApJ, 715, 202

Hopkins P. F. et al., 2010b, ApJ, 724, 915

Huang K.-H. et al., 2017, ApJ, 838, 6

Huertas-Company M. et al., 2013, MNRAS, 428, 1715

Huertas-Company M. et al., 2016, MNRAS, 462, 4495

Jiang F. et al., 2019, MNRAS, 488, 4801

Jiménez-Andrade E. F. et al., 2019, A\&A, 625, A114

Kawamata R., Ishigaki M., Shimasaku K., Oguri M., Ouchi M., 2015, ApJ, 804, 103

Kawinwanichakij L. et al., 2020, ApJ, 892, 7

Kocevski D. D. et al., 2017, ApJ, 846, 112

Kravtsov A. V., 2013, ApJ, 764, L31

Kravtsov A. V., Vikhlinin A. A., Meshcheryakov A. V., 2018, Astron. Lett., 44,8

Kubo M., Yamada T., Ichikawa T., Kajisawa M., Matsuda Y., Tanaka I., Umehata H., 2017, MNRAS, 469, 2235

Lapi A. et al., 2011, ApJ, 742, 24

Lapi A. et al., 2018a, ApJ, 857, 22

Lapi A., Salucci P., Danese L., 2018b, ApJ, 859, 2

Leauthaud A. et al., 2012, ApJ, 744, 159

Leja J., Speagle J. S., Johnson B. D., Conroy C., van Dokkum P., Franx M., 2020, ApJ, 893, 111

Lower S., Narayanan D., Leja J., Johnson B. D., Conroy C., Davé R., 2020, ApJ, 904, 33

Luo Y. et al., 2020, MNRAS, 493, 1686

Lustig P. et al., 2021, MNRAS, 501, 2659

McDermid R. M. et al., 2015, MNRAS, 448, 3484

McLeod D. J., McLure R. J., Dunlop J. S., Cullen F., Carnall A. C., Duncan K., 2021, MNRAS, 503, 4413

Man A. W. S., Zirm A. W., Toft S., 2016, ApJ, 830, 89

Mandelbaum R., Wang W., Zu Y., White S., Henriques B., More S., 2016, MNRAS, 457, 3200

Mantha K. B. et al., 2018, MNRAS, 475, 1549

Martig M., Bournaud F., Teyssier R., Dekel A., 2009, ApJ, 707, 250

Matthee J., Schaye J., Crain R. A., Schaller M., Bower R., Theuns T., 2017, MNRAS, 465, 2381

Meert A., Vikram V., Bernardi M., 2015, MNRAS, 446, 3943

Meert A., Vikram V., Bernardi M., 2016, MNRAS, 455, 2440

Miller T. B., van Dokkum P., Mowla L., van der Wel A., 2019, ApJ, 872, L14
Mo H. J., Mao S., White S. D. M., 1998, MNRAS, 295, 319

Mo H., van den Bosch F. C., White S., 2010, Galaxy Formation and Evolution. Cambridge Univ. Press, Cambridge

Mosleh M., Tacchella S., Renzini A., Carollo C. M., Molaeinezhad A., Onodera M., Khosroshahi H. G., Lilly S., 2017, ApJ, 837, 2

Moster B. P., Naab T., White S. D. M., 2013, MNRAS, 428, 3121

Moster B. P., Naab T., White S. D. M., 2018, MNRAS, 477, 1822

Mowla L., van der Wel A., van Dokkum P., Miller T. B., 2019a, ApJ, 872, L13

Mowla L. et al., 2019b, ApJ, 880, 57

Mundy C. J., Conselice C. J., Duncan K. J., Almaini O., Häußler B., Hartley W. G., 2017, MNRAS, 470, 3507

Muzzin A. et al., 2013, ApJ, 777, 18

Naab T., Johansson P. H., Ostriker J. P., 2009, ApJ, 699, L178

Navarro J. F., Frenk C. S., White S. D. M., 1996, ApJ, 462, 563

Newman A. B., Ellis R. S., Bundy K., Treu T., 2012, ApJ, 746, 162

Nipoti C., Treu T., Auger M. W., Bolton A. S., 2009, ApJ, 706, L86

Nipoti C., Treu T., Leauthaud A., Bundy K., Newman A. B., Auger M. W., 2012, MNRAS, 422, 1714

O'Leary J. A., Moster B. P., Naab T., Somerville R. S., 2021, MNRAS, 501, 3215

Oser L., Ostriker J. P., Naab T., Johansson P. H., Burkert A., 2010, ApJ, 725, 2312

Oser L., Naab T., Ostriker J. P., Johansson P. H., 2012, ApJ, 744, 63

Patel S. G., Hong Y. X., Quadri R. F., Holden B. P., Williams R. J., 2017, ApJ, 839, 127

Peng Y.-j. et al., 2010, ApJ, 721, 193

Pillepich A. et al., 2018, MNRAS, 473, 4077

Poggianti B. M., Moretti A., Calvi R., D’Onofrio M., Valentinuzzi T., Fritz J., Renzini A., 2013, ApJ, 777, 125

Posti L., Fall S. M., 2021, A\&A, 649, A119

Puglisi A. et al., 2019, ApJ, 877, L23

Puglisi A. et al., 2021, preprint (arXiv:2103.12035)

Qu Y. et al., 2017, MNRAS, 464, 1659

Ragone-Figueroa C., Granato G. L., 2011, MNRAS, 414, 3690

Ribeiro B. et al., 2016, A\&A, 593, A22

Rodriguez-Gomez V. et al., 2015, MNRAS, 449, 49

Rodríguez-Puebla A., Avila-Reese V., Drory N., 2013, ApJ, 767, 92

Rodríguez-Puebla A., Avila-Reese V., Yang X., Foucaud S., Drory N., Jing Y. P., 2015, ApJ, 799, 130

Rodríguez-Puebla A., Primack J. R., Avila-Reese V., Faber S. M., 2017, MNRAS, 470, 651

Saglia R. P. et al., 2010, A\&A, 524, A6

Sánchez Almeida J., 2020, MNRAS, 495, 78

Saracco P., Longhetti M., Gargiulo A., 2010, MNRAS, 408, L21

Shankar F., Bernardi M., 2009, MNRAS, 396, L76

Shankar F., Lapi A., Salucci P., De Zotti G., Danese L., 2006, ApJ, 643, 14

Shankar F., Marulli F., Bernardi M., Boylan-Kolchin M., Dai X., Khochfar S., 2010, MNRAS, 405, 948

Shankar F., Marulli F., Bernardi M., Mei S., Meert A., Vikram V., 2013, MNRAS, 428, 109

Shankar F. et al., 2014, ApJ, 797, L27

Shankar F. et al., 2015, ApJ, 802, 73

Shankar F. et al., 2017, ApJ, 840, 34

Sherman S. et al., 2020, MNRAS, 499, 4239

Shibuya T., Ouchi M., Harikane Y., 2015, ApJS, 219, 15

Smail I. et al., 2021, MNRAS, 502, 3426

Sohn J., Geller M. J., Diaferio A., Rines K. J., 2020, ApJ, 891, 129

Somerville R. S., Davé R., 2015, ARA\&A, 53, 51

Somerville R. S. et al., 2018, MNRAS, 473, 2714

Sparre M., Springel V., 2016, MNRAS, 462, 2418

Stockmann M. et al., 2021, ApJ, 908, 135

Stringer M. J., Shankar F., Novak G. S., Huertas-Company M., Combes F., Moster B. P., 2014, MNRAS, 441, 1570 (S14)

Suess K. A., Kriek M., Price S. H., Barro G., 2019, ApJ, 877, 103

Suess K. A., Kriek M., Price S. H., Barro G., 2020, ApJ, 899, L26 
Sun F. et al., 2021, ApJ, 908, 192

Tacchella S., Dekel A., Carollo C. M., Ceverino D., DeGraf C., Lapiner S., Mandelker N., Primack J. R., 2016, MNRAS, 458, 242

Tacchella S., Carollo C. M., Faber S. M., Cibinel A., Dekel A., Koo D. C., Renzini A., Woo J., 2017, ApJ, 844, L1

Tacchella S. et al., 2019, MNRAS, 487, 5416

Tadaki K.-i. et al., 2020, ApJ, 901, 74

Thomas D., Maraston C., Bender R., Mendes de Oliveira C., 2005, ApJ, 621, 673

Tinker J. L., 2017, MNRAS, 467, 3533

Tinker J. L. et al., 2017, ApJ, 839, 121

Tortora C. et al., 2018, MNRAS, 481, 4728

Trujillo I., Conselice C. J., Bundy K., Cooper M. C., Eisenhardt P., Ellis R. S., 2007, MNRAS, 382, 109

Trujillo I., Ferreras I., de La Rosa I. G., 2011, MNRAS, 415, 3903

Trujillo I., Chamba N., Knapen J. H., 2020, MNRAS, 493, 87

Vale A., Ostriker J. P., 2006, MNRAS, 371, 1173

van den Bosch F. C., Jiang F., Hearin A., Campbell D., Watson D., Padmanabhan N., 2014, MNRAS, 445, 1713

van der Vlugt D., Costa T., 2019, MNRAS, 490, 4918

van der Wel A. et al., 2014, ApJ, 788, 28

van Dokkum P. G., Franx M., 1996, MNRAS, 281, 985

van Dokkum P. G. et al., 2010, ApJ, 709, 1018

van Dokkum P. G. et al., 2015, ApJ, 813, 23

Wang T. et al., 2019, Nature, 572, 211

Wechsler R. H., Tinker J. L., 2018, ARA\&A, 56, 435

Whitney A., Conselice C. J., Bhatawdekar R., Duncan K., 2019, ApJ, 887, 113

Wilman D. J., Fontanot F., De Lucia G., Erwin P., Monaco P., 2013, MNRAS, 433, 2986

Zanisi L. et al., 2020, MNRAS, 492, 1671 (Z20)

Zhou L. et al., 2020, A\&A, 642, A155

Zolotov A. et al., 2015, MNRAS, 450, 2327

\section{SUPPORTING INFORMATION}

Supplementary data are available at MNRAS online.

Figure S5. The number density of CSFMGs (cyan) and CQMGs (red) for Model 1 (top row) and Model 2 (bottom row).

Please note: Oxford University Press is not responsible for the content or functionality of any supporting materials supplied by the authors. Any queries (other than missing material) should be directed to the corresponding author for the article.

\section{APPENDIX A: THE $f_{\text {QUENCH }}(z)$ RELATION}

In Fig. A1, we show the evolution of $f_{\text {Quench }}$ from equations (5) and (6) for $\mu=1,3,5$. It can be seen that in models with a higher $\mu$ the halo mass scale above which galaxies are statistically quenched evolves much faster with redshift, and is higher at earlier cosmic times.

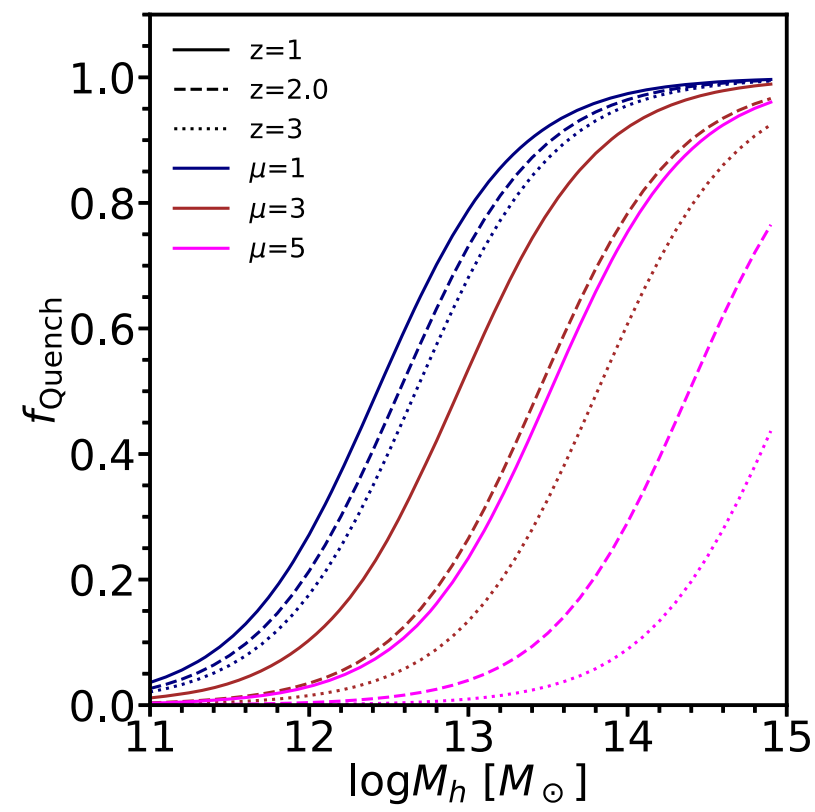

Figure A1. The evolution of $f_{\text {Quench }}$ from equations (5) and (6) for $\mu=5$ (magenta lines) $\mu=3$ (red lines) and for $\mu=1$ (blue lines). In both cases, dotted lines, dashed lines, and solid lines are for $z=3,2,1$, respectively. It can be seen that in models with a higher $\mu$ the halo mass scale above which galaxies are statistically quenched evolves much faster with redshift, and is higher at earlier cosmic times.

\section{APPENDIX B: CALIBRATION OF $A_{K}$}

We use Sloan Digital Sky Survey DR7 data (Abazajian et al. 2009) to calibrate $A_{K, S F}$ and $A_{K, Q}$ at $z \sim 0.1$. We create a mock catalogue of MGs at $z=0.1$ as detailed in Section 2. We then constrain the normalization $A_{K}$ for star-forming and quiescent galaxies by matching the mean size of the MGs in our mock catalogue to the mean observed semimajor axis effective radius that best fits the light profiles of Massive Late Type Galaxies (LTGs) and Early Type Galaxies (ETGs) from the Meert et al. (2015), Meert, Vikram \& Bernardi (2016), Domínguez Sánchez et al. (2018) r-band photometric and morphological catalogues. We define LTGs as those objects for which TType $>0$ and ETGs those that have TType $\leq 0$. We assume that all massive LTGs are star-forming and all massive ETGs are quiescent. The light profile is truncated as in Fischer, Bernardi \& Meert (2017).

\section{APPENDIX C: DEFINITIONS OF COMPACTNESS}

In Fig. C1, we show the number density evolution of compact quenched and CSFMGs, for different definitions of compactness (including the one adopted in the main text of this paper, that is, that of Cassata et al. 2011), and for Model 1. 

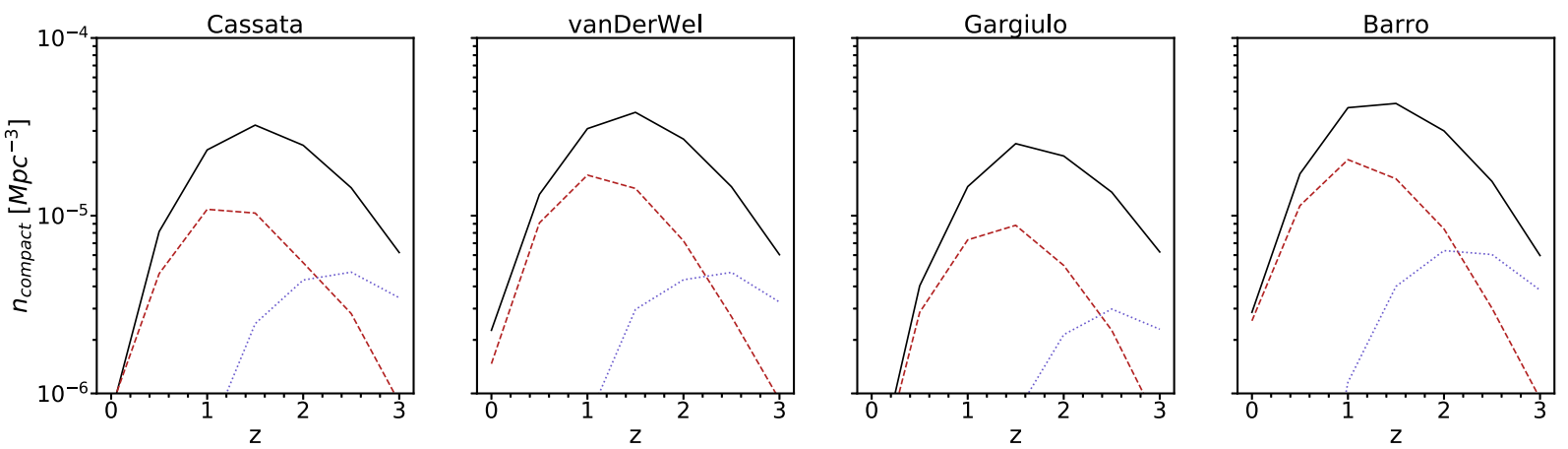

Figure C1. The evolution of the number density of compact quenched (red dashed lines) and compact star-forming (blue dotted lines) MGs, for Model 1 and $\mu=3$, as in Fig. 4. Compactness is defined as in Cassata et al. (2011) (left), van der Wel et al. (2014) (centre left), Gargiulo et al. (2017) (centre right), and Barro et al. (2013) (right). Distinct definitions of compactness yield qualitatively very similar results, although quantitatively different.

\section{APPENDIX D: COMPACTS IN MODELS 3 AND 4}

Fig. D1 shows the evolution of the number density of MGs and the corresponding predictions for the numbers of CQMGs and CSFMGs for Models 3 and 4. The results are qualitatively (but not quantitatively) similar to Models 1 and 2 (see Fig. 4). In particular, most quenching models seem to disagree with the current data for star-forming galaxies.
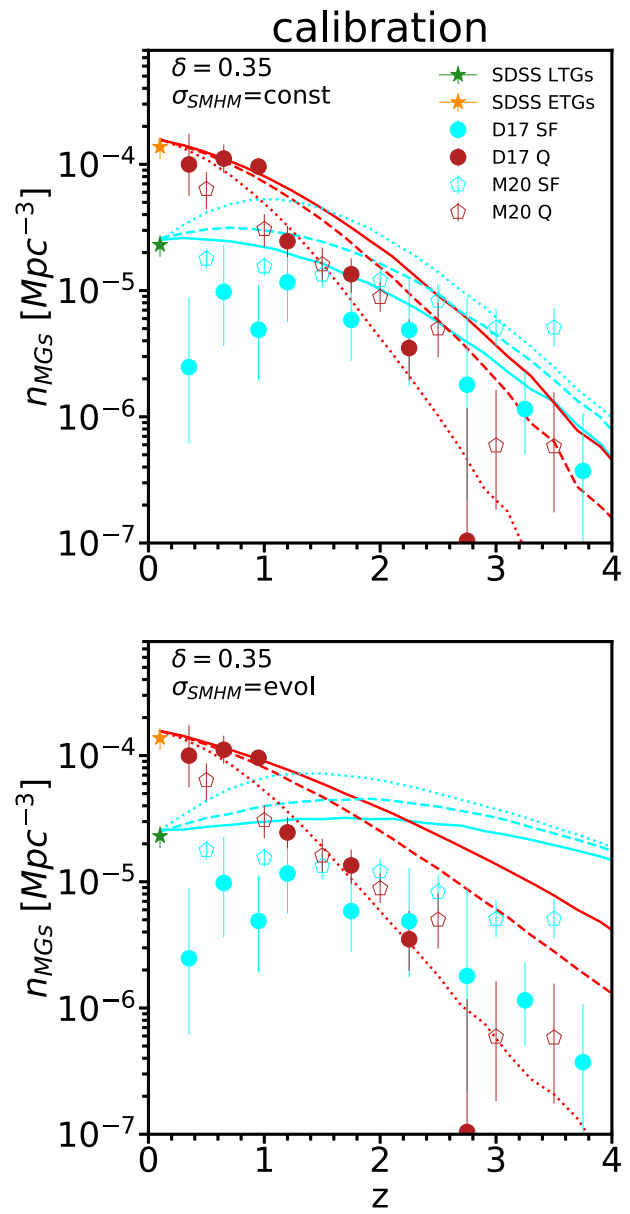

\section{APPENDIX E: USING OTHER SIZE ESTIMATORS}

Recent works have explored different definitions for the size of a galaxy. For instance, Mowla et al. (2019a) and Miller et al. (2019) put forward the idea that the radius that encloses 80 per cent of the light, $R_{80}$, might be more fundamental than $R_{e}$. This claim is based on the observation that (i) the size distributions of star-forming and
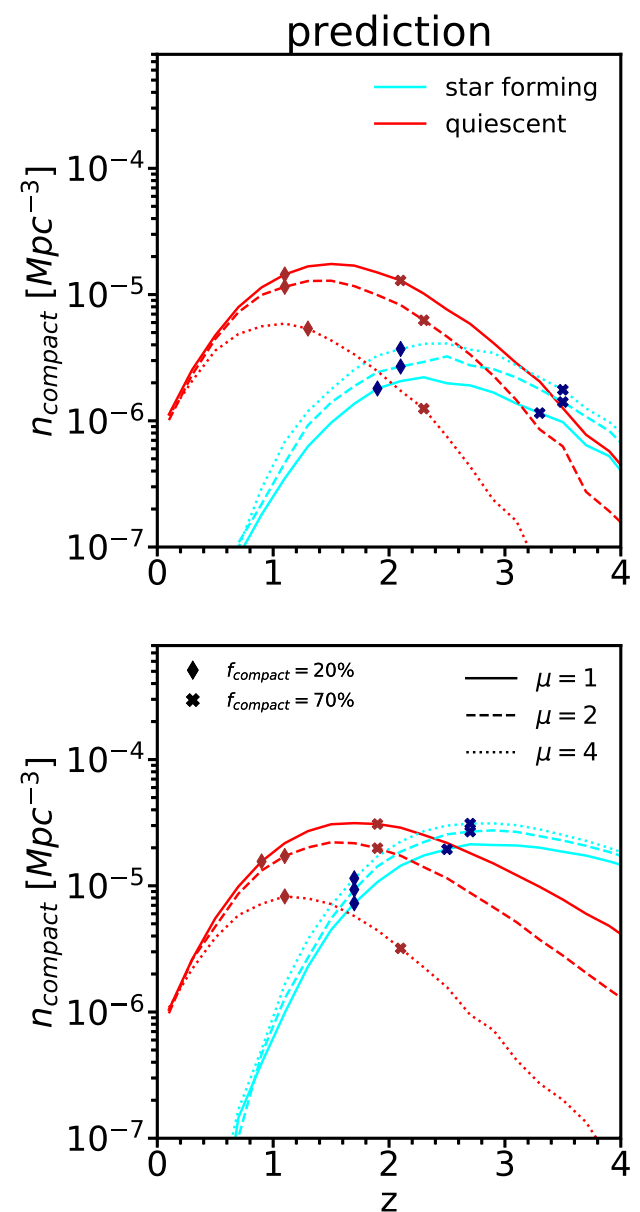

Figure D1. Left: The number density of star-forming and quenched MGs (cyan and red line, respectively) for models 3 and 4 . Solid, dashed, and dotted lines are obtained adopting $\mu=1,2$, and 4, respectively. Right: Prediction for the number density of compact MGs for the two models and the different values of $\mu$. The comparison data in the left column are from the SDSS 'cmodel' photometry at $z=0.1$, and Davidzon et al. (2017), McLeod et al. (2021) (not corrected for the $\mathrm{M} / \mathrm{L}$ as it was done in Fig. 4, see Grylls et al. 2020b) at higher redshift. 
quenched galaxies are almost identical when using $R_{80}$ as opposed to the use of $R_{\mathrm{e}}$, and (ii) that the shape and evolution of the $R_{80}-M_{\text {star }}$ is reminiscent of the SMHM relation. Although we make explicit mention of $R_{\mathrm{e}}$ throughout this paper, our model can be used to make predictions for the size evolution and the number density evolution of compact MGs, regardless of star formation activity, which may be interpreted in terms of $R_{80}$, rather than $R_{\mathrm{e}}$. In particular, the K13 model would read

$$
R_{80}=A_{K, 80} R_{\mathrm{h}}
$$

Using the values of $R_{80}$ for SDSS that we computed in Z20, we find that that for MGs $\left\langle R_{80}\right\rangle \approx 4.2\left\langle R_{\mathrm{e}}\right\rangle$. Therefore, we expect $A_{K, 80} \approx$ 4.2A .

Trujillo et al. (2020) and Chamba, Trujillo \& Knapen (2020) used deep imaging of local galaxies to define $R_{1}$ as the radius that encloses the region within a physically motivated mass surface density of $1 \mathrm{M}_{\odot} \mathrm{pc}^{-2}$ (see also Sánchez Almeida 2020). Trujillo et al. (2020) found that the scatter in the $R_{1}-M_{\text {star }}$ relation is of the order of only $\approx 0.06$ dex across five orders of magnitude, including the regime of MGs for which the relation, which is linear at lower masses, breaks. The $R_{1}-R_{\mathrm{h}}$ relation would read

$$
R_{1}=A_{K, 1} R_{\mathrm{h}} \text {. }
$$

Using the publicly available catalogue of $R_{1}$ measurements from Trujillo et al. (2020) and Chamba et al. (2020), we find that $\left\langle R_{1}\right\rangle \approx$ $7.8\left\langle R_{\mathrm{e}}\right\rangle$, which implies that the normalization of the $\mathrm{K} 13$ relation in equation (E2), $A_{K, 1} \approx 7.8 A_{K}$. In Section 3.4 (Fig. 3), we have shown that using a constant value of $A_{K}$ works remarkably well to describe the evolution of $R_{\mathrm{e}}$. Whether this will be the case also for $R_{1}$ will be revealed by future deep high-redshift observations.

Lastly, we would like to highlight the fact that different size definitions provide different pieces of information: while $R_{e}$ is tight to the concentration of the light profile (see Chamba et al. 2020), $R_{80}$ probes also the outer regions of the galaxy. Likewise, $R_{1}$ has been proposed based on the gas mass density threshold required to initiate star formation. We believe that this does not necessarily make a definition of size more fundamental than another. Thus, it is possible that distinct definitions of galaxy sizes may be related to different physical processes generated by distinct galaxy-halo coevolution paths.

This paper has been typeset from a $\mathrm{T}_{\mathrm{E}} \mathrm{X} / \mathrm{L} \mathrm{T}_{\mathrm{E}} \mathrm{X}$ file prepared by the author. 